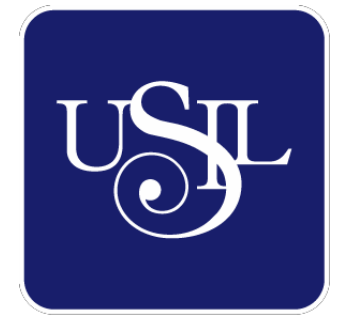

UNIVERSIDAD

SAN IGNACIO

DE LOYOLA

FACULTAD DE HUMANIDADES

Carrera de Psicología

\title{
INFLUENCIA DE LOS RASGOS DE PERSONALIDAD SOBRE EL CONSUMO DE ALCOHOL EN ESTUDIANTES UNIVERSITARIOS DE LIMA EN ÉPOCA DE PANDEMIA POR LA COVID - 19
}

Tesis para optar el Título Profesional de Licenciado en Psicología

\author{
NATALI PAOLA ARRASCO FERNÁNDEZ \\ (0000-0002-1424-0588)
}

\author{
Asesor: \\ Dr. Sergio Alexis Dominguez Lara \\ (0000-0002-2083-4278) \\ Lima - Perú \\ 2021
}




\section{Agradecimiento}

A mi familia y a todas las personas que brindaron su apoyo en la realización de esta investigación. 


\section{Resumen}

Los estudiantes universitarios son una población vulnerable y propensa al consumo de alcohol debido a distintos factores que permiten esta actividad e influyen dentro su entorno. Por esta razón, la presente investigación tuvo como finalidad determinar la influencia de los rasgos de personalidad sobre el consumo de alcohol en estudiantes universitarios de Lima. La muestra estuvo conformada por 207 estudiantes universitarios (64\% mujeres) con edades comprendidas entre 18 y 29 años $(\mathrm{M}=21.9, \mathrm{DE}=2.26)$. Se empleó el Cuestionario de Identificación de los Trastornos debidos al Consumo de Alcohol (AUDIT) el cual evalúa los patrones de consumo de riesgo, perjudicial y dependiente de alcohol y el Cuestionario de Personalidad de Zuckerman-Kuhlman-Aluja Personality Questionnaire Shortened Form (ZKA-PQ/SF) el cual mide los rasgos búsqueda de sensaciones, extraversión, neuroticismo, actividad y agresividad. Los resultados evidenciaron la influencia significativa y positiva de extraversión $(\beta=.305)$, neuroticismo $(\beta=0.156)$ y actividad $(\beta=.182)$ sobre el consumo de alcohol. Se concluyó que algunos rasgos de personalidad influyen significativamente sobre el consumo de alcohol.

Palabras clave: Consumo de alcohol, estudiantes universitarios, rasgos de personalidad. 


\begin{abstract}
University students are a vulnerable population prone to alcohol consumption due to different factors that allow this activity and influence their environment. For this reason, the present research aimed to determine the influence of personality traits on alcohol consumption in university students from Lima. The sample consisted of 207 university students (64\% women) aged between 18 and 29 years $(\mathrm{M}=21.9, \mathrm{SD}=2.26)$. The Alcohol Consumption Disorders Identification Questionnaire (AUDIT) was used, which assesses risky, harmful and alcoholdependent consumption patterns and the Zuckerman - Kuhlman - Aluja Personality Questionnaire Shortened Form (ZKA- PQ / SF) which measures sensation seeking, extraversion, neuroticism, activity and aggressiveness traits. The results showed the significant and positive influence of extraversion $(\beta=.305)$, neuroticism $(\beta=0.156)$ and activity $(\beta=$ .182) on alcohol consumption. It was concluded that some personality traits significantly influence alcohol consumption.
\end{abstract}

Keywords: Alcohol consumption, personality traits, university students 


\section{Introducción}

En el Perú, se calcula que la ingesta anual de bebidas alcohólicas es de 6.3 litros de alcohol puro per cápita en la población de mayores de 15 años, esta cantidad ubica al país en el noveno lugar de Latinoamérica que, a su vez, junto a Norte América y Centro América, es la segunda región con mayor consumo de alcohol en el mundo, siendo consumidora el 54,1\% de su población (Organización Mundial de la Salud [OMS], 2018). Asimismo, la OMS (2018) indica que el Perú se encuentra entre los cuatro países en Sudamérica que presenta mayor índice de prevalencia de consumo excesivo de alcohol (49.5\%) en personas mayores de 15 años. Cabe resaltar que, en el Perú, el consumo de alcohol en mujeres mayores de 15 años es de 2.2 litros per cápita, mientras que el consumo de hombres, desde la misma edad, es de 10.4 litros per cápita (OMS, 2018); se ha identificado que usualmente los hombres ingieren alcohol en mayor medida y con mayor frecuencia que las mujeres (OMS, 2018; Rada \& Ispas, 2016; Ruisoto, Cacho, López-Goni, Vacab \& Jiménez, 2016), asimismo, que existe una cantidad más amplia de mensajes permisivos sobre consumo de alcohol dirigidos a hombres que a mujeres, y a su vez, estas últimas son mayormente juzgadas al realizar esta conducta (Rada \& Ispas, 2016).

Por otro lado, la OMS indica que se espera un aumento de la conducta de consumo de alcohol global per cápita en los próximos 6 años debido a la predisposición existente actual al consumo. Un estudio del Ministerio de Salud del Perú (MINSA, 2017) aseveró que el consumo de alcohol en estudiantes universitarios peruanos obtiene una prevalencia de vida del $87.1 \%$, siendo esto considerablemente alto.

A pesar de que el consumo de alcohol puede suponer una práctica que provee placer a los usuarios, la realización de esta conducta de manera recurrente puede llegar a ser peligroso y causar un notable daño en el individuo (Babor, Higgins-Biddle, Saunders \& Monteiro, 2001). 
Babor et al. (2001) identifican que el consumo de alcohol puede ser de bajo riesgo, de riesgo, nocivo, y de dependencia. La OMS (1994) describe el consumo de riesgo como un patrón de ingesta de alcohol el cual conlleva al individuo a una situación de posibles consecuencias adversas y posible daño; dicho consumidor no presenta ningún síndrome ni síntoma, ni ha experimentado daño físico ni psicológico, sin embargo, esta conducta representa la exposición al peligro. El consumo nocivo, también llamado consumo excesivo de alcohol (Herring et al. 2008), hace referencia al patrón de ingesta de alcohol de manera desmedida que presenta consecuencias negativas o daños en términos de salud física, mental y social con respecto al usuario y está asociado a la causa de una amplia gama de daño hacia los individuos, en caso de accidentes, y a la comunidad, en el caso del crimen y desorden.

La dependencia, por otro lado, también llamados adicción al alcohol o alcoholismo (OMS, 1994) hace referencia a una enfermedad en la cual existen las siguientes manifestaciones comportamentales, cognitivas y fisiológicas: Consumo repetitivo de alcohol, de manera que el usuario, en este caso llamado adicto, se intoxica de forma habitual y continua, indicando un deseo compulsivo de adquirir la sustancia, demuestra una exacerbada dificultad para dejar de consumir voluntariamente o cambiar el consumo de alcohol y manifiesta decisión por obtener la sustancia por cualquier medio. Generalmente, la persona manifiesta tolerancia a la sustancia, lo que quiere decir que la respuesta que en un inicio generaba a consecuencia del consumo de una dosis determinada, se ve disminuida debido al uso continuo, asimismo para alcanzar dicha respuesta inicial, la persona se ve obligada a consumir dosis más altas cada vez (OMS, 1994). De igual manera, La OMS (1994) indica que se presenta síndrome de abstinencia el cual se caracteriza por ser un conjunto de síntomas intensos que aparecen al interrumpir la ingesta de la sustancia al haber sido consumida de forma repetitiva, durante largos periodos de tiempo y en altas dosis, mismo que ocurre al momento de suspender el consumo. En este caso, la vida de esta persona se encuentra subyugada por la ingesta de alcohol, y excluye 
generalmente todas las demás actividades y responsabilidades que podría llegar a tener (OMS, 1994).

Según la OMS (2018) el consumo de alcohol es el origen de más de 200 enfermedades y trastornos, de igual manera se encuentra ligada al desarrollo de relaciones conflictivas con amigos, familia y allegados, abandono de responsabilidades, así como posibles accidentes domésticos, de tráfico y laborales.

Los jóvenes estudiantes universitarios comprenden un grupo vulnerable frente al consumo de alcohol debido a diversos factores que influyen dentro de este entorno en particular (Valdivia-Lívano et al., 2018) como la adaptación a nuevos y diferentes círculos sociales, la exposición ante un ambiente más tolerante frente al consumo de sustancias psicoactivas, la facilidad para obtener bebidas alcohólicas en cuanto al horario variado de estudios así como la falta de supervisión de los padres o apoderados, la presión social o el hecho de querer agradar al grupo de pares (Londoño, García, Valencia \& Vinaccia, 2005), así como el estrés generado por la transición de la adolescencia a la adultez y los estudios universitarios (Deasy, Coughlan, Pironom, Jourdan, \& Mcnamara, 2014). En general, el consumo de alcohol aumenta en jóvenes al momento de ingresar a la universidad (Cameron et al., 2015). Por ejemplo, en el Perú, Silva (2020) determinó que en el 2015 existió mayor prevalencia de problemas relacionados al consumo de alcohol en los estudiantes universitarios pertenecientes a los tres primeros años de carrera de la Universidad Mayor de San Marcos. Asimismo, la estadía de los estudiantes en la universidad es un factor por el cual dicho consumo se puede volver excesivo (Jobnston, O'Malley, Bacbman, \& Schulenberg, 2004). Se ha determinado que los jóvenes quienes no viven con sus padres o pareja son quienes consumen alcohol en mayor medida y frecuencia, por lo que podrían verse vulnerables quienes dejan de vivir con sus familias por ir a estudiar a la universidad (Rada e Ispas, 2016). De igual manera, diversos estudios (Hunt \& Eisenberg, 2010; Kassa et al., 2016) concluyeron que el consumo problemático de alcohol contribuye a 
que los estudiantes se vean envueltos en conductas sexuales de riesgo, a la pobre ejecución de funciones, al bajo rendimiento académico y a que dichos estudiantes sean menos propensos a buscar ayuda en caso de presentar problemas de salud mental (Mekonen et al., 2017).

En ese sentido, la literatura e investigación se ha enfocado en determinar los factores de riesgo para el consumo de alcohol entre universitarios, los cuales señalan ser sociodemográficos, de desarrollo, psicosociales, ambientales, contextuales, así como de personalidad (Cicognani \& Zani, 2014).

Diferentes rasgos de personalidad han sido asociados con el consumo de alcohol (Adan, Navarro \& Forero, 2016; Cicognani \& Zani, 2014; Littlefield \& Sher, 2010; Motos et al., 2015; Pilatti, Urrizaga, Chincolla \& Cupani, 2014) y han sido demostrados de predecir dicho consumo (Skeel, Pilarski, Pytlak \& Neudecker, 2008). De este modo, rasgos como búsqueda de sensaciones, impulsividad, extraversión y neuroticismo explican parcialmente la tendencia de susceptibilidad frente al uso de sustancias psicoactivas y la posibilidad de que dicho consumo pueda caer en alguna de las categorías ya sea consumo de riesgo, nocivo o de dependencia (Marquez-Arrico \& Adan, 2013), así como también la existencia de patologías duales, las cuales son comorbilidades entre un trastorno de uso de sustancias y trastorno mental como esquizofrenia, depresión, ansiedad, etc.

\section{El modelo de los cinco factores alternativos de Zuckerman (M5FA)}

El M5FA surge como una disyuntiva frente al modelo de tres factores de Eysenck (1967), el modelo de los 16 factores de Catell (1957) y el modelo de cinco factores de Costa y McCrae (1992) (Zuckerman, Kullman, Joireman, Teta \& Kraft, 1993).

El modelo de Zuckerman toma elementos del sistema de personalidad de Eysenck, en primer lugar, ambos son modelos psicobiológicos los cuales miden los rasgos de extraversión 
y neuroticismo, sin embargo, el M5FA deja de lado la variable psicoticismo y la reemplaza por la de búsqueda de sensaciones y agresividad (Zuckerman \& Cloninger, 1996).

En cuanto al sistema de los 16 factores de Cattell (1957), Zuckerman considera que se trata de un modelo muy extenso, el cual podría comprimirse entre sus factores y, asimismo, lo critica por considerarlo un modelo no replicable en cuanto a género, edad y métodos (Zuckerman et al., 1993).

Por otro lado, el autor del M5FA indica que el modelo de los Cinco Grandes Factores de Costa y McCrae, no cuenta, en su totalidad, con factores con bases biológicas y asevera que los rasgos de personalidad deben ser estudiados a nivel biológico desde lo comportamental hasta lo genético (Zuckerman, 1992).

Los cinco factores alternativos según el modelo de Zuckerman (2002) se dividen en búsqueda de sensaciones, extraversión, neuroticismo, agresividad, actividad. Con respecto al rasgo búsqueda de sensaciones-impulsividad, más adelante nombrado solo "búsqueda de sensaciones" (Aluja, Kuhlman \& Zuckerman, 2010), Zuckerman (2002) afirma que existe una obvia relevancia entre este factor y la toma de riesgos. Asimismo, lo define como la búsqueda de sensaciones y experiencias variadas, novedosas, complejas e intensas, y la disposición a tomar riesgos físicos, sociales, legales y financieros con tal de realizar dichas experiencias, donde existe la susceptibilidad al aburrimiento y la desinhibición (Zuckerman, 2002). Cabe resaltar la definición que le da a impulsividad como la tendencia a involucrarse en situaciones o rápidamente responder ante ciertas señales que devienen en posibles recompensas, sin necesariamente haberlo planeado o deliberado y sin tomar en cuenta algún posible castigo o pérdida de recompensa; por otro lado, lo considera como la incapacidad de inhibición del peligro de la conducta "búsqueda de recompensa". En ese sentido, da a conocer que este rasgo de personalidad ha sido relacionado con la participación en distintas actividades de riesgo como 
experimentos de posible riesgo, deportes, vocación, actividades criminales conducta sexual, fumar, consumo excesivo de alcohol, abuso de drogas, conducir auto habiendo consumido psicoactivos, entre otros (Zuckerman, 2002). Por otro lado, Ruch y Zuckerman (2001) afirman que la toma de riesgo, o el ponerse en riesgo, no es el objetivo principal de la búsqueda de sensaciones; ejemplifica que las personas con un grado menor del rasgo no están simplemente evitando el riesgo, sino que no encuentran recompensa en la sensación o experiencia que podría justificar este. Es importante mencionar que, entre sus investigaciones, Zuckerman (1994) encontró que la diferencia prominente entre factores demográficos relacionados al rasgo búsqueda de sensaciones, fue que este es mayor en hombres que en mujeres, que aumenta entre las edades 9 a 14 años, alcanza su pico máximo en la adolescencia tardía o en los 20s y disminuye de manera continua conforme la edad va aumentando. Adicional a esto, Crane, Gorka, Phana y Childs (2018) investigaron la relación entre búsqueda de sensaciones y la actividad cerebral; afirmaron que, y en concordancia con la literatura, existe una relación significativa entre la conectividad de la amígdala derecha - corteza frontal orbitaria, la conectividad de la amígdala inferior derecha- corteza frontal orbitaria y la búsqueda de sensaciones, asimismo, las alteraciones en esta zona del cerebro se encuentran relacionadas con características propias del rasgo como impulsividad, déficit en la regulación de emociones, autocontrol y la toma de riesgos (White, 2017).

En cuanto al rasgo extraversión, se vincula con las emociones positivas, las personas con alto nivel de extraversión se muestran como comprometidas, quienes se motivan rápido, tienen una actitud de apertura frente a la acción, sobre todo si involucra recompensa (White, 2017), muestran sociabilidad, es decir, disfrutan de la interacción afectiva, valoran los lazos estrechos interpersonales, son cordiales, prefieren estar en compañía que solos, disfrutan de las fiestas o las reuniones grandes (Lucas, Diener, Grob, Suh \& Shao, 2000), asimismo, poseen tendencia al cumplimiento de objetivos, dominio social, generalmente son líderes, 
exhibicionistas y tienden a ser asertivos (Depue \& Collins, 1999). Schmidt (2010) explica que las personas con alta puntuación en extraversión poseen bajos niveles en el circuito retículo cortical, por lo que, al obtener mayor estimulación ambiental pueden regular de manera adecuada la activación cortical, y esto explicaría el por qué dichas personas tienden a buscar energía en el entorno; sin embargo, no es la única estructura cerebral que explica el rasgo.

En cuanto al rasgo neuroticismo, Zuckerman (2002) lo describe como la propensión a experimentar tensión, inseguridad, preocupación, miedo, en general, sentimientos negativos, así como hostilidad, depresión, timidez y vulnerabilidad (Dyce, 1997). Personas con trastornos mentales suelen presentar el rasgo en mayor nivel (Dash, Slutske, Martin, Statham, Agrawal \& Lynskey, 2019; Marquez-Arrico \& Adan, 2013); Kotov, Gamez, Schmidt y Watson (2010) lo describen como el rasgo de personalidad predictor de patologías por excelencia y Dyce (1997), como el "sello" de los trastornos de personalidad. Asimismo, un estudio realizado a través de 22 países indicó que neuroticismo se dio en mayor nivel en mujeres que en hombres en todos los países (Kajonius \& Giolla, 2017). Por otro lado, Zuckerman (1992) vinculó la sintomatología ansiosa con actividad cerebral en la amígdala principalmente. Schmidt (2010) indica que la amígdala "posee proyecciones hacia otras regiones del cerebro (por ejemplo, el hipotálamo) implicadas en las reacciones de miedo y ansiedad. También existen proyecciones que conectan la amígdala con áreas del córtex, especialmente con los lóbulos frontal y temporal" (p. 22).

Zuckerman (2002) define que el rasgo agresividad refleja la tendencia a expresarse de forma irascible y ruda, las personas con alto nivel del rasgo se muestran hostiles, desafiantes, desconsiderados, descorteses, impacientes y poseen baja deseabilidad social. Según la literatura, Zuckerman y Khuleman determinaron que este rasgo también se encuentra relacionado positivamente con conductas de riesgo, algunas de estas fueron dependencia al alcohol, ofensas violentas, conducta sexual de riesgo y hábitos de manejo inadecuados. En un 
estudio de Huang et al. (2011), los resultados determinaron que el rasgo predice los trastornos de personalidad paranoide y narcisista, asimismo, que pacientes pertenecientes al Cluster B (trastornos de personalidad antisocial, narcisista, histriónico y límite) poseen niveles altos de agresividad. Además, en un estudio realizado por Aluja et al. (2019) en 18 países, se determinó que los hombres tienden a mostrar mayor agresividad que las mujeres y se encuentra en mayor nivel en hombres mayores de 46 años.

Finalmente, el autor describe que las personas con mayor nivel del rasgo actividad son enérgicos, se encuentran en constante movimiento, muestran interés por las actividades que requieren esfuerzo, reflejan incomodidad al no tener nada que hacer por lo que tienen una necesidad de mantenerse activos (Zuckerman, 2002). Según Aluja et al. (2019), actividad en general incrementa a partir de los 30 años y se mantiene; sin embargo, en mujeres, disminuye a partir de los 60 años. En este mismo estudio (Aluja et al. 2019), se determinó que las mujeres muestran mayor nivel del rasgo en el grupo de 18 a 30 años.

El M5FA es un modelo psicobiológico, cuyo principal aporte en investigación y entornos clínicos es su capacidad de predecir la vulnerabilidad a los trastornos mentales (Escorial et al. 2015). Por ejemplo, en un estudio realizado por Aluja et al. (2007) se modelo demostró ser un buen predictor de los trastornos de personalidad del DSM y en particular, del Cluster B (trastorno de personalidad antisocial, narcisista, límite e histriónico). Asimismo, suele describir de manera más adecuada los rasgos de personalidad tendientes a la disfuncionalidad (Huang et al. 2011).

En cuanto a rasgos de personalidad que facilitan el consumo de alcohol en estudiantes universitarios, estudios relevantes indican que el rasgo búsqueda de sensaciones (Adan, Forero \& Navarro, 2017; Cicognani \& Zani, 2014; Hosier \& Cox, 2011; Jones, Chryssanthakis \& Groom, 2014; Loxton et al., 2015) suele ser el mejor predictor. De igual manera, el rasgo 
extraversión (Adan et al., 2017, Hakulinen et al., 2015; Turiano et al., 2012). Zuckerman (2000) afirma que el consumo de bebidas alcohólicas, al comenzar generalmente en fiestas, es mayormente disfrutado por personas extrovertidas y buscadoras de sensaciones. Por una parte, Jones et al. (2014) indican que las personas con mayor nivel del rasgo búsqueda de sensaciones buscarían consumir más cantidad de alcohol con el objetivo de añadir novedad a las situaciones de manera que se tornen variadas, complejas e intensas y se pueda repotenciar la sensación de excitación, incluso cuando las consecuencias sean negativas. Por otro lado, en cuanto a extraversión, Adams et al. (2012), refiere que el consumo de alcohol se da para la obtención de una recompensa social, la cual sería diversión entre amigos, desarrollar mayor sociabilidad, disfrutar de la reunión, entre otros, esto reforzaría positivamente la ingesta de alcohol en aquellos con mayor nivel del rasgo, lo cual, a su vez, se relaciona a un mayor consumo por su parte.

En otras investigaciones, el rasgo que se encuentra relacionado positivamente, de igual manera, principalmente es el neuroticismo (Adan et al., 2017; Hicks et al., 2012, Kotov, 2010; Turiano et al., 2012). Por ejemplo, se encontró que la sintomatología ansiosa y depresiva en estudiantes universitarios se asoció con mayor consumo de alcohol, mayor frecuencia de consumo excesivo y mayores consecuencias negativas por el consumo (Adan et al., 2017, Hakulinen et al., 2015, Turiano et al., 2012). Adams et al., (2012) indican que aquellos individuos quienes lidian con mayor estrés, ansiedad y, en general, emocionas negativas, tienden a consumir alcohol de manera urgente como mecanismo de afrontamiento en búsqueda de alivio momentáneo e incremento o facilitación de un estado de ánimo positivo.

Identificar los estudios realizados que relacionan rasgos de personalidad con el consumo de alcohol en la población de jóvenes estudiantes universitarios es fundamental para el entendimiento del vínculo existente entre dichas variables en contextos tanto 
latinoamericanos como extranjeros. Asimismo, para poder extrapolar dicho conocimiento al entorno peruano.

Poo y Montes (2010) llevaron a cabo un estudio con objetivo de analizar la relación entre rasgos de personalidad y patrones de consumo de alcohol en jóvenes. La muestra estuvo constituida por 247 adultos entre 18 y 29 años de edad de la ciudad de Mar del Plata. El 59 \% fueron hombres, el 98\% había completado el nivel primario de educación. Se utilizó el cuestionario " $Z K P Q-50-C C$ " el cual fue creado por Aluja et al. (2006) y está compuesto por cinco escalas: agresión-hostilidad, impulsividad-búsqueda de sensaciones, neuroticismoansiedad, sociabilidad y activación. Realizaron un cuestionario empleando el método de cantidad-frecuencia con preguntas sobre la frecuencia de la ingesta de alcohol, la elección de bebida y la cantidad de tragos consumidos; se estimó cuatro patrones de consumo de alcohol: consumo moderado o bajo, consumo alto, consumo episódico excesivo y consumo episódico no excesivo. Los resultados demostraron que únicamente la variable búsqueda impulsiva de sensaciones se encontró asociada con el patrón de consumo excesivo [OR=2,53 (95\% CI: 1,052,07), $p<0.05]$. Respecto al patrón de consumo elevado se determinaron asociaciones para el rasgo neuroticismo $[\mathrm{OR}=0,47$ (95\% CI: 0,25-0,82), $p<0.05]$, para el rasgo actividad $[\mathrm{OR}=0,39$ (95\% CI: 0,21-0,75), $p<0.01]$ y para el rasgo búsqueda impulsiva de sensaciones $[\mathrm{OR}=3,07$ (95\% CI: 1,6-5,8), $p<0.01]$. Se concluyó que la variable búsqueda impulsiva de sensaciones se mostró asociada al consumo alto de bebidas alcohólicas y al consumo excesivo episódico. Por otra parte, la variable extraversión, y bajos puntajes del rasgo neuroticismo se mostraron asociados a una ingesta de alcohol menor.

Adan, Navarro y Forero (2016) realizaron un estudio con el objetivo de reconocer el perfil de personalidad de los estudiantes universitarios consumidores de alcohol excesivo. La muestra estuvo conformada por 140 estudiantes universitarios españoles, entre 19 y 25 años y 60 eran hombres. Un grupo de 70 estudiantes eran consumidores de alcohol excesivo y el otro 
grupo era de control. Los estudiantes no habían tenido historia de dependencia de alcohol o cualquier otra droga, historia familiar de alcoholismo, consumo de droga frecuente ni contaban con enfermedades mentales. Para medir personalidad, aplicaron el instrumento ZKPQ (Gomài-Freixanet et al., 2004) el cual cuenta con 5 dimensiones: neuroticismo y ansiedad, extraversión, búsqueda de sensaciones e impulsividad, agresividad y actividad. Los resultados arrojaron que la dimensión de personalidad neuroticismo influyó de manera significativa entre el grupo de consumo excesivo de alcohol y el grupo control a favor del grupo inicial $\left(\eta^{2} \mathrm{p}=\right.$ 0.103); del mismo modo en la dimensión de Impulsividad y búsqueda de sensaciones se hayo influencia significativa en la cual el grupo de consumo excesivo de alcohol puntuaba más alto $\left(\eta_{\mathrm{p}}^{2}=0.295\right)$. Se concluyó que los programas de intervención deberían mediar los factores de riesgo de personalidad.

Palacios (2018) llevó a cabo un estudio cuyo objetivo fue la validez factorial del consumo de alcohol, a partir de la búsqueda de sensaciones. La muestra estuvo conformada por 550 jóvenes entre 14 y 23 años, de la Ciudad de México y 290 fueron mujeres. Se empleó un cuestionario el cual incluía 4 preguntas: La edad de inicio de consumo de alcohol, frecuencia de consumo de alcohol en el último mes (nunca - diario), la cantidad de alcohol consumido por evento (número de vasos consumidos por ocasión y la incidencia en cuanto a consumo excesivo de alcohol (5 o más vasos por evento) y número de vasos los cuales ellos consideran que necesitan para embriagarse. Para medir búsqueda de sensaciones, se utilizó el Inventario Mexicano de Búsqueda de sensaciones creado por Palacios (2015) la cual cuenta con 8 subescalas: búsqueda de placer, búsqueda de riesgos, búsqueda de emociones intensas, búsqueda de novedades, búsqueda de emociones y aventuras, desinhibición, búsqueda inusual y susceptibilidad al aburrimiento. Los resultados arrojaron que la búsqueda de sensaciones es significativamente un predictor de la conducta de consumo de alcohol $(\beta=.37, p<.05)$. Concluyen que, si los individuos mantienen un nivel alto en cuanto a "búsqueda de 
sensaciones", el consumo de alcohol aumentará; asimismo, el nivel de búsqueda de sensaciones aumentará mediante la cantidad de estimulación que dichos individuos experimentan.

Cicognani y Zani (2014) plantearon una investigación con el objetivo de evaluar el rol de la búsqueda de sensaciones, consumo de alcohol entre grupos de estudiantes universitarios y la autoeficacia para resistir el consumo frente a la presión de grupo. Se empleó una muestra de 588 estudiantes universitarios entre 19 y 26 años, de 6 facultades distintas de una universidad en el norte de Italia. El 27\% estuvo conformado por hombres, el 20.2\% vivía en la misma ciudad con sus familias, 30.3\% vivían en las afueras de la ciudad (dentro de los $100 \mathrm{~km}$ ) y con sus familias, $49.5 \%$ venían de otras partes de Europa y no vivían con sus familias. El consumo de alcohol fue evaluado a través de un cuestionario el cual medía frecuencia de consumo de cuatro bebidas alcohólicas diferentes, dentro de la cual, un ítem medía la frecuencia de episodios de ebriedad durante los 30 anteriores días y otro ítem medía la frecuencia de consumo entre grupo de pares. La Búsqueda de sensaciones fue medida a través de la escala "Sensation Seeking (impss)" del cuestionario "Zuckerman-Kuhlman Personality Questionnaire (ZKPQ)" (Zuckerman, Kuhlman, Thomquist, \& Kiers, 1991). Asimismo, la autoeficacia fue medida a través de un item cuán capaces se sentían de resistirse a la presión de grupo al momento de beber alcohol. Como resultado se obtuvo que la búsqueda de sensaciones obtuvo una correlación positiva contundente con la frecuencia de consumo de alcohol $(r=.43, p<.001)$ y con la frecuencia de episodios de ebriedad $(r=.42, p<.001)$, asimismo, la frecuencia de consumo de alcohol entre amigos percibida fue también significativa y positivamente correlacional con la frecuencia de consumo $(r=.48, p<.001) \mathrm{y}$ con frecuencia de episodios de ebriedad $(r=.40, p<.001)$. Concluyeron que es importante la intervención preventiva orientadas a normas de grupos y mejorar sus habilidades de rechazo.

Pilatti, Urrizaga, Chincolla y Cupani (2014) realizaron un estudio con el objetivo de investigar de qué manera las facetas de personalidad se relacionan con diferentes modalidades 
de consumo de alcohol en estudiantes universitarios de Córdoba (Argentina). La muestra estuvo conformada por 400 estudiantes universitarios de entre 17 y 58 años de edad, el $20.8 \%$ de los participantes cursaba primer año, el 17\% cursaba segundo, el 33.3\% cursaba tercero, el $13.8 \%$ cursaba cuarto, el $14.8 \%$ cursaba quinto, el $0.3 \%$ cursaba sexto y un participante $(0.3 \%)$ no aportó información del año de cursado. Se utilizó el "Inventario de Personalidad NEOIPIP" (Goldberg, 1999) con 5 factores de personalidad: Neuroticismo, Extraversión, Responsabilidad, Amabilidad y Apertura. Para medir consumo de alcohol se empleó el "Cuestionario de Consumo de Alcohol” el cual evalúa frecuencia y cantidad de consumo y clasifica en consumo ligero a moderado, consumo episódico elevado, consumo riesgoso. Los resultados determinaron que las facetas que presentan utilidad discriminativa son inmoderación (Neuroticismo) y búsqueda de emociones (Extraversión) vinculándola así con el grupo de consumidores riesgosos. Se concluyó que los resultados hallados fundamentan la elaboración de una escala para la evaluación de las características de personalidad asociadas al consumo de alcohol.

Pilatti, Cupani y Pautassi (2015) ejecutaron una investigación con el objetivo de conocer patrones de consumo de alcohol en una muestra de universitarias argentinas según rasgos de personalidad y las expectativas de consumo de alcohol. La muestra estuvo conformada por 298 mujeres estudiantes de primer año universitario, pertenecientes a diferentes cursos en el Departamento de Psicología de la Universidad Nacional. Se empleó una encuesta para determinar consumo de alcohol, la cual medía frecuencia de consumo, bebida consumida y cantidad. Para medir rasgos de personalidad se empleó la versión en español del Big five questionnaire for children $(B F Q-C)$ con los factores: Neuroticismo, Extraversión, Responsabilidad, Amabilidad y Apertura. Los resultados indicaron que el rasgo Extraversión estuvo significativamente asociado con el grupo de bebedores regulares de consumo excesivo quienes puntuaron más alto que el grupo de abstemios $(\mathrm{OR}=1.20)$, de igual manera con el 
grupo de consumidores moderados, quienes puntuaron más alto que el grupo de abstemios (OR $=1.14$ ) y también con el grupo de bebedores regulares de consumo excesivo, quienes puntuaron más alto que el grupo de consumidores moderados $(\mathrm{OR}=1.05)$. En cuanto al rasgo de Búsqueda de experiencias, este resultó significativamente relacionado con el grupo de bebedores regulares de consumo excesivo en comparación con el grupo de consumidores moderados $(\mathrm{OR}=2.50)$. Concluyeron que se puede diferenciar el grupo de estudiantes universitarias con consumo excesivo de alcohol del grupo de consumo moderado.

Motos, Cortés, Giménez y Cadaveira (2015) realizaron una investigación con el propósito de analizar el rol de la personalidad y edad de inicio en el consumo de alcohol en la predicción del consumo semanal de alcohol, y variables en las consecuencias asociadas al consumo en jóvenes universitarios. La muestra estuvo con 213 consumidores intensivos de primer curso de la Universidad Complutense de Madrid. El 56.8\% fueron mujeres y la edad media fue de 18.20. Para evaluar el consumo de alcohol, se registró la edad de inicio de consumo y el patrón de consumo Se utilizó el Inventario de Personalidad Reducido NEO-FFI de Costa y McCrae (1999) para medir rasgos de personalidad; el inventario BIS11 de Barratt (Patton et al., 1995) para medir Impulsividad con tres variables: Impulsividad Cognitiva, Motora y No planificada; asimismo, se empleó la escala de Consecuencias del instrumento IECI (Cortés et al., 2012) para dictaminar referencia a diferentes consecuencias que cada joven afirma estar experimentando debido a su consumo, las cuales se describen en: síntomas físicos, pérdida del control, conductas de riesgo, dependencia física y autopercepción. Los resultados determinaron que existe una correlación nula entre consumo de alcohol y neuroticismo ( $\mathrm{r}=-.04$; p>.05) y extraversión $(r=.078 ; p>.05)$, y, por otro lado, una correlación positiva entre consumo de alcohol con el rasgo de Impulsividad $(r=.185, p<.01)$. De igual manera, se obtuvo una correlación positiva y significativa entre consecuencias experimentadas y extraversión $(r=.135 ; p<.05)$, neuroticismo $(r=.154 ; p<.05)$ e impulsividad $(r=.310 ; p<$ 
.01). Se concluyó que los rasgos de impulsividad y responsabilidad explican en gran parte la conducta de consumo semanal entre varones y que las consecuencias derivadas del consumo resultan explicativas a través de la impulsividad y neuroticismo.

El estudio realizado por Jones, Chryssanthakis y Groom (2014) tuvo como objetivo evaluar la interrelación entre consumo de alcohol, impulsividad, motivos del consumo y tendencia a participar en conductas problemáticas relacionadas con el alcohol. La muestra estuvo conformada por un total de 400 estudiantes entre 18 y 25 años, de los cuales 148 fueron hombres. El consumo de alcohol fue medido a través del instrumento "Student Alcohol Questionnaire Revised" (SAQ; Engs \& Hanson, 1994) el cual mide frecuencia de consumo, cantidad, bebida alcohólica consumida y consecuencias negativas ocurridas debido al consumo; este último ítem midió la participación en conductas problemáticas relacionadas con el consumo. Los motivos de consumo fueron medidos a través de la escala "Drinking Motivation Questionnaire” (DMQ; Cooper, 1994), la cual midió los siguientes factores: motivos de intensificación, motivos sociales, motivos de conformidad y motivos de mecanismo de afrontamiento. Impulsividad fue medida a través del instrumento "UPPS Impulsive Behaviour Scale" (Whiteside \& Lynam, 2003), la cual evalúa cuatro factores: urgencia, falta de premeditación, falta de perseverancia y búsqueda de sensaciones. Los resultados arrojaron que la búsqueda de sensaciones produce efectos significativos sobre la cantidad de alcohol consumida: relación con cerveza $(r=.25 ; p<.001)$, relación con vino $(r=.27 ; p=<.001) \mathrm{y}$ relación con spirits $(r=.24 ; p<.001)$.

De igual manera, Hakulinen et al. (2015) realizaron un estudio con el objetivo de evaluar el rol de la personalidad como determinante para el consumo de alcohol. Se realizó un metaanálisis de una muestra de 72,949 personas de Estados unidos, Alemania, Australia y Reino Unido. Contó con una media de 50 años y el $54 \%$ fueron mujeres. Se emplearon diferentes instrumentos orientados a la medición de los Cinco Grandes Factores (Goldberg, 
1999): Extraversión, Neuroticismo, Amabilidad, Apertura y Responsabilidad. Los resultados arrojaron que el consumo excesivo de alcohol estuvo asociado con una alta extraversión (OR $1.14 ; 1.09-1.20)$ y alto neuroticismo (OR $1.20 ; 95 \%$ CI 0.83-0.96). En conclusión, los hallazgos sugieren que consumo de alcohol alto y en incremento es más común en extrovertidos y aquellos con baja responsabilidad, mientras que una alta amabilidad y poca apertura a la experiencia pueden aumentar las probabilidades de que las personas reduzcan el consumo de alcohol y prefieran la abstinencia.

Por su parte, González-Iglesias, Gómez-Fraguela, Gras y Planes (2014) analizaron la relación entre la búsqueda de sensaciones y el consumo de alcohol en jóvenes. La muestra estuvo comprendida por 356 jóvenes con edades comprendidas entre los 18 y los 25 años. El 62.4\% fueron mujeres, todos eran estudiantes de Psicología de las Universidades de Santiago de Compostela y Girona. Se usó el Inventario de Búsqueda de sensaciones de Arnett (1994) la cual cuenta con las siguientes subescalas: Intensidad y Novedad. Para evaluar el abuso del alcohol utilizamos la versión breve del Alcohol Use Disorders Test-Consumption Questionnaire (AUDIT-C) (Bush, Kivlahan, McDonell \& Bradley, 1998). Los resultados obtenidos confirman que la búsqueda de experiencias intensas y novedosas predice de forma importante el consumo de alcohol $(\beta=.17 ; p=.001)$ y este se correlaciona positiva significativamente con experiencias intensas $(r=.32 ; p<.01)$ y novedosas $(r=.3 ; p<.01)$. Concluyeron que la búsqueda de sensaciones es importante para explicar el consumo de alcohol de los jóvenes.

Asimismo, Nogueira-Arjona, et al. (2019) ejecutaron un estudio donde examinaron el impacto del rasgo de personalidad "extraversión" y el nivel de consumo de alcohol en acompañamiento social en estudiantes universitarios. La muestra estuvo conformada por 298 estudiantes universitarios, es decir, 149 “compañeros de bebida" estudiantes universitarios. Cada pareja estuvo conformada por compañeros de bebida del mismo sexo, con quienes no 
mantuvieran relaciones románticas, haberse conocido hace un año o menos, tuvieran acceso a internet en casa, que incluyeran al menos un estudiante universitario de primer año, tuvieran entre 18 y 25 años de edad, y hubieran bebido al menos 12 bebidas alcohólicas en el último año. Se empleó el instrumento "Big Five Inventory" (BFI; John \& Srivastava, 1999) para medir el rasgo extraversión. Para medir el nivel de consumo de alcohol y contextos sociales de consumo de alcohol, se empleó el instrumento Self-Administered Timeline Followback (STLFB; Collins, Kashdan, Koutsky, Morsheimer \& Vetter, 2008). Los resultados evidenciaron que la extraversión moderó positivamente el consumo de alcohol de los compañeros de bebida, en acompañamiento, es decir que la interacción entre el alto nivel de bebida estando acompañado y la extraversión fue significativa. Se pudo comprobar la relación de la extraversión con el alto consumo de alcohol en compañía de otra persona.

Valdivia-Lívano et al. (2018) realizaron una investigación que tuvo como objetivo principal determinar la frecuencia y los factores asociados al consumo alcohólico de riesgo en estudiantes universitarios de la ciudad de Huancayo. La muestra estuvo conformada por 293 estudiantes de medicina de 3 universidades de Huancayo. El 54\% fueron mujeres, todos los participantes estuvieron entre los 18 y 35 años. Se utilizó el cuestionario "AUDIT (Test de identificación de los Trastornos debidos al Consumo de Alcohol)" creado por la OMS y para medir la presencia de síntomas depresivos utilizaron el Inventario de Depresión de Beck (Beck, Ward, Mendelson, Mock \& Erbaugh, 1961) el cual clasifica los síntomas depresivos en cuatro niveles: ausente, leve, moderado y severo. Los resultados evidencian asociación entre el sexo femenino y mayor consumo riesgoso de alcohol (RPc: 1,13; IC 95\%: 1,01-1,28). El análisis multivariado determinó, de igual manera, la relación entre el análisis bivariado y el sexo femenino (RPa: 1,15; IC 95\%: 1,10-1,19; valor $p<0,001$ ); así como la asociación con facultades de ciencias de la salud frente a humanidades (RPa: 0,34; IC 95\%: 0,13-0,90; valor $p=0,030)$ y la asociación entre consumo riesgoso de alcohol y la presencia de síntomas 
depresivos (RPa: 2,45; IC95\%: 2,18-2,76; valor $p<0,001$ ). Concluyeron que gran cantidad de estudiantes presentaron alcoholismo, y estuvo asociado al sexo femenino, con facultades de ciencias de la salud frente a humanidades y la presencia de síntomas depresivos.

A modo de resumen, los antecedentes propuestos describen la relación entre rasgos de personalidad y consumo de alcohol en estudiantes universitarios. Cada investigación emplea como mínimo dos instrumentos psicométricos a fin de evaluar cada variable. La limitación más importante que se observa es la escasez de estudios en el medio peruano relacionando las dos variables en la muestra propuesta. Adicional a esto, se evidencia que solo dos investigaciones emplean el instrumento diseñado bajo una teoría de personalidad psicobiológica y una de ellas, no es un estudio dirigido a estudiantes universitarios, sino, a jóvenes en general. Si bien los estudios miden la relación entre personalidad y consumo de alcohol en estudiantes universitarios, estos lo hacen principalmente tomando en cuenta el modelo de los cinco grandes factores, por lo que sólo se tienen datos de relación entre las variables extraversión, neuroticismo y búsqueda de sensaciones, en la mayoría de los casos.

Por todo lo expuesto con anterioridad, se elabora la siguiente pregunta de investigación: ¿Existe influencia significativa de los rasgos de personalidad sobre el consumo de alcohol en estudiantes universitarios de Lima?, de este modo, se aborda como objetivo general determinar de qué manera influyen los rasgos de personalidad sobre el consumo de alcohol en estudiantes universitarios de Lima. A partir de ello, se proponen los siguientes objetivos específicos:

- Determinar cómo influye la búsqueda de sensaciones sobre el consumo de alcohol

- Determinar cómo influye la extraversión sobre el consumo de alcohol

- Determinar cómo influye el neuroticismo sobre el consumo de alcohol

- Determinar cómo influye la agresividad sobre el consumo de alcohol 
- Determinar cómo influye la actividad sobre el consumo de alcohol

Este estudio se justifica debido a que frente a los cambios en el estilo de vida generados por la pandemia del COVID-19 los estudiantes pueden verse en la posibilidad de adoptar mecanismos negativos de afrontamiento (Son, Hegde, Smith, Wang \& Sasangohar, 2020) lo cual incluiría un incremento en el consumo de alcohol.

La pandemia del COVID 19 exigió que el gobierno tomara distintas medidas y proporcionara restricciones a la población para evitar la propagación del virus. Una de las cuales fue el aislamiento social obligatorio el cual empezó el 15 de marzo del 2020 (“Coronavirus: Perú decreta cuarentena", 2020) y concluyó el 30 de junio ("Perú levanta cuarentena", 2020), lo cual conllevó a la inmovilización obligatoria y suspensión de actividades, dentro de ellas, el cierre temporal de universidades ("Decreto Supremo", 2020). Esto implicó que la gente modificara su estilo de vida y dentro de estos cambios se vio un incremento en la inactividad y sedentarismo, así como mayor consumo de comida poco saludable (Ammar et al., 2020). En cuanto al cambio del estilo de vida en universitarios, estos presentaron incremento en sedentarismo (Romero-Blanco et al, 2020) reducción de la actividad física (Gallè et al., 2020), incremento en sintomatología ansiosa y depresiva, lo cual involucró dificultad de concentración, discontinuidad en los patrones de sueño, disminución de las interacciones sociales debido al distanciamiento, preocupación en la salud y preocupación por el rendimiento académico (Son et al., 2020).

Por otra parta, si bien en el Perú existen investigaciones realizadas sobre la relación entre rasgos de personalidad y consumo de alcohol en estudiantes universitarios, estas son escasas (Arana, 2013), asimismo, no existen estudios que midan relación entre consumo de alcohol y rasgos de personalidad bajo un modelo M5FA. Escorial et al. (2015) indica que los modelos biológicos de la personalidad son importantes ya que "tienen gran mayor utilidad en 
entornos clínicos y de investigación, dado su mayor potencial para desarrollar hipótesis de trabajo sobre los correlatos biológicos y conductuales, así como para predecir la vulnerabilidad a los trastornos mentales" (p.20). Se han realizado distintas investigaciones desde un punto de vista clínico empleando el modelo M5FA las cuales han sido de gran aporte para la literatura científica (Ball, 2004; Ramos-Grille et al., 2015; Sáez-Francàs et al.,2014; Valero et al., 2014).

En ese sentido, los resultados beneficiarán la evaluación y futura comparación de estudios afines (Poo \& Montes, 2010), de igual manera, se podrá evidenciar la necesidad de planes de intervención por parte de las universidades, a partir de un enfoque integral hacia aquellos estudiantes con un perfil propenso al consumo de alcohol de riesgo.

Frente a la investigación, se proponen las siguientes hipótesis específicas:

1. Los rasgos búsqueda de sensaciones, extraversión y neuroticismo influirán significativa y positivamente sobre el consumo de alcohol en estudiantes universitarios.

2. Los rasgos agresividad y actividad no influirán de manera significativa sobre el consumo de alcohol en estudiantes universitarios. 


\section{Método}

\section{Tipo y diseño de investigación}

El presente estudio se estableció dentro de un diseño predictivo transversal (Ato, López \& Benavente, 2013) ya que se investigó la manera en la que la variable rasgos de personalidad pronostica a la variable consumo de alcohol en los estudiantes universitarios.

\section{Participantes}

La muestra estuvo conformada por 207 estudiantes universitarios de Lima Metropolitana donde el 64\% fueron mujeres y el 36\% fueron hombres, todos los participantes de 18 a 29 años $(M=21.9, D E=2.26)$, del primer al décimo ciclo, quienes ingresaron a la universidad entre el año 2014 y el 2020, donde el 86\% residía en Lima y el 14\% fuera de Lima, en su mayoría solteros $99 \%$, el $88 \%$ pertenecientes a una universidad privada y $12 \%$ a una universidad pública. Como criterio de inclusión se consideró que los estudiantes consumieran alcohol en algún grado, fueran de nacionalidad peruana, pertenecieran a una universidad peruana y que fueran jóvenes entre 18 a 29 años. Como criterio de exclusión se consideró si los estudiantes habían recibido tratamiento psiquiátrico o se encontraban actualmente en uno, si eran extranjeros o si tenían edades fuera del rango indicado.

El tamaño de la muestra fue establecido en base a correlaciones $(r)$ vinculadas al análisis estadístico de regresión que se realizó empleando el software G*Power 3.1.7 (Faul, Erdfelder, Lang \& Buchner, 2007), y con un poder estadístico de potencia .95 , con una correlación esperada de $r=.2255$, se determinó que la muestra mínima necesaria fue 204 estudiantes.

\section{Instrumento}


Se empleó El Cuestionario de Identificación de los Trastornos debidos al Consumo de Alcohol (AUDIT) el cual fue creado por la OMS y publicado en 1989. Tuvo una actualización en 1992 y más adelante fue adaptada al español por Rubio, Bermejo, Caballero y SantoDomingo (1998) y adaptado en el Perú por Rafael Aramburú (2017). El instrumento estima, por medio de 10 ítems tipo Likert, los patrones de consumo de riesgo, perjudicial y dependiente de alcohol. En cuanto a su división, el grupo de las tres primeras preguntas indican el uso de bebidas alcohólicas, las siguientes cuatro preguntas aluden a la dependencia y las últimas se refieren a las consecuencias negativas. El cuestionario se puntúa del 0 a 40 y un puntaje igual o mayor a 8 (hombres) y 7 (mujeres) refleja un consumo de riesgo y perjudicial. A mayor puntaje, mayor es la probabilidad de que se dé un consumo problemático (Babor et al., 2001).

El instrumento fue validado y adaptado por Aramburú (2017) quien realizó la adaptación lingüística de la prueba al medio peruano y evidenció la validez de la estructura interna a través de un análisis factorial exploratorio. Respecto a sus propiedades de confiabilidad, el estudio indica una alta confiabilidad evaluada por el Coeficiente Alfa de Cronbach con valores de .80 en estudiantes universitarios de Lima (Aramburú, 2017).

Asimismo, se utilizó el Cuestionario de Personalidad de Zuckerman-Kuhlman-Aluja Personality Questionnaire Shortened Form (ZKA-PQ/SF) (Aluja et al., 2019). El cuestionario está conformado por 80 ítems, el cual se encuentra adaptado al español por Aluja et al. (2019), y apto para su uso en Latinoamérica, incluyendo Perú (Aluja, 2019; comunicación personal), cuya consistencia interna, evaluada por el Coeficiente Alfa de Cronbach, presenta valores mayores a .77. La escala mide cinco dimensiones básicas de la personalidad y cada dimensión se evalúa a través de 20 facetas y 4 ítems por faceta: búsqueda de sensaciones, que mide la intención de participar y vivir experiencias que incluyan algún riesgo físico, social, legal o financiero; extraversión, la cual define a una persona sociable y optimista; neuroticismo, describe la tendencia hacia la preocupación emocional, el miedo, la indecisión y la obsesión; 
agresividad, hace referencia a la expresión de forma belicosa; y actividad, referente al agrado por mantenerse ocupado o en movimiento.

\section{Procedimiento}

Se elaboró un protocolo de evaluación a modo de encuesta a través de Google Forms el cual incluyó el consentimiento informado, apartado que detalla el objetivo de la investigación, el anonimato y confidencialidad de los datos a tratar con el único fin de la investigación, la ficha sociodemográfica y las dos escalas, el cual contó con una duración aproximada de 15 minutos.

Inicialmente, se contactó a las autoridades correspondientes según lo señalado por cada institución y se explicó, a través de correo electrónico, acerca de la investigación propuesta, así como su carácter de confidencialidad y anonimato, y se solicitó la colaboración de la universidad. Sin embargo, debido a la baja tasa de respuesta obtenida por correo institucional, se decidió optar por la distribución de la encuesta a través de redes sociales a los estudiantes de distintas universidades, por lo que no fue necesario pedir permiso a ninguna institución, no obstante, se requirió el uso del consentimiento informado y la aceptación de este por parte de los estudiantes previamente al acceso y desarrollo del cuestionario. Se realizó la publicación de la encuesta en las siguientes redes sociales: Facebook, Twitter e Instagram en conjunto con un sorteo de 27.75 dólares, el cual se empleó como incentivo para incrementar la participación de los estudiantes universitarios. Una vez terminado el plazo para la recolección de información, se procedió a excluir aquellas encuestas cuyos participantes no cumplían con los criterios de inclusión.

\section{Plan de análisis}

Respecto al análisis estadístico, se realizó el análisis descriptivo correspondiente de los datos. Seguido de esto, se estableció la confiabilidad a través del coeficiente de $\alpha$ y la 
correlación inter-ítem promedio (rii) sobre cada área, cuyos valores fueron aceptados al encontrarse el $\alpha$ alrededor de .60 y rii en el rango de .15 a .20 al ser el (ZKA-PQ/SF) un instrumento que evalúa constructos amplios (Clark \& Watson, 1995).

Previo al análisis de regresión, se realizó la evaluación de los supuestos de normalidad de asimetría y curtosis para lo cual se empleó el límite de 3; de linealidad por medio de la correlación de Pearson, la cual identifica la magnitud del efecto adecuado cuando $r>.20$; de independencia de errores, se muestra aceptable cuando el valor del estadístico de Watson resulta entre 1.5 y 2.5 ; de multicolinealidad, se muestra aceptable cuando resulta mayor a .10 y el factor de inflación de varianza resulta menor a 10, e indica ausencia de la misma (Ferguson, 2009).

Con el objetivo de determinar la existencia de influencia de rasgos de personalidad sobre consumo de alcohol, a continuación, se emplearon dos estadísticos enfocados en la regresión múltiple: el $R^{2}$ que cuantifica la variabilidad general de los predictores, el cual se valora como insignificante cuando es menor a .04 , baja cuando resulta entre .04 y .25 , moderada entre .25 y .64 y fuerte cuando es mayor a .64; y el coeficiente beta estandarizado ( $\beta$ ), el cual cuantifica la influencia individual de los predictores, se valora según la magnitud del efecto y es insignificante cuando resulta menor a .20 , baja entre .20 y .50 , moderada entre .50 y .80 y fuerte cuando es mayor a .80 (Ferguson, 2009).

Para la realización de los análisis estadísticos previamente descritos, se empleó el software Jamovi (The Jamovi Project, 2020). 


\section{Resultados}

\section{Análisis descriptivos y de confiabilidad}

Los indicadores de confiabilidad ( $\alpha$ y rii) fueron excelentes para Consumo de alcohol $(\alpha=.803 ;$ rii $=.29)$ y adecuado para extraversión $(\alpha=.673 . ;$ rii $=.114)$, neuroticismo $(\alpha=.783$; rii $=.184)$, agresividad $(\alpha=.717 ;$ rii $=.184)$ y actividad $(\alpha=.725 ;$ rii $=.141)$. Sin embargo, la magnitud de búsqueda de sensaciones $(\alpha=0.587 . ;$ rii $=0.08)$ no fue la mínima aceptable, por lo cual se decidió prescindir de la variable.

\section{Tabla 1}

Análisis descriptivo de los rasgos de las variables del estudio

\begin{tabular}{lcccccc}
\hline & $M$ & $D E$ & $g 1$ & $g 2$ & $\alpha$ & rii \\
\hline Consumo de alcohol & 6.92 & 5.45 & .992 & .670 & .803 & .29 \\
Búsqueda de & 43.7 & 4.98 & -.127 & -.222 & .587 & .82 \\
sensaciones & & & & & & \\
Extraversión & 42.2 & 5.09 & -.218 & .134 & .673 & .114 \\
Neuroticismo & 37.7 & 6.81 & -.0229 & -.142 & .783 & .184 \\
Agresividad & 36.3 & 6.16 & .04 & -.97 & .717 & .137 \\
Actividad & 37.4 & 5.52 & .150 & -.284 & .725 & .141 \\
\hline
\end{tabular}

Nota: $\mathrm{M}=$ Media; $\mathrm{DE}=$ desviación estándar; g1= coeficiente de asimetría de Fisher; g2= coeficiente de curtosis de Fisher; $\alpha=$ alfa de Cronbach; rii= correlación inter-ítem promedio

\section{Evaluación de los supuestos}

Con respecto a la evaluación de los supuestos, se aprecia un acercamiento a la normalidad evidenciado en las magnitudes de asimetría $(<1)$ y curtosis $(<1)$ (Tabla 1$)$. Se encontró correlación significativa entre el consumo de alcohol y extraversión $(\mathrm{r}=.307 ; \mathrm{p}<$ $.001)$ y consumo de alcohol y actividad $(r=.290 ; p<.001)$, por otro lado, se halló la correlación entre consumo de alcohol y neuroticismo ( $r=.138 ; p=.048)$, consumo de alcohol y agresividad $(r=.105 ; p=.134)$. Asimismo, se muestra el estadístico de Durbin-Watson aceptable (2.08) y 
ausencia de multicolinealidad en vista de los valores para extraversión (tolerancia $=.911$; FIV $=1.10)$, neuroticismo $($ tolerancia $.727 ;$ FIV $=1.38)$, agresividad $($ tolerancia $=.713 ;$ FIV $=1.40)$ $\mathrm{y}$ actividad (tolerancia $=.909 ; \mathrm{FIV}=1.10)$.

\section{Contraste de hipótesis}

Con relación a las hipótesis (Tabla 2), se evidencia la influencia global de magnitud baja de los rasgos de personalidad sobre el consumo de alcohol. Se brinda respaldo empírico a la primera hipótesis especifica ya que se aprecia que los rasgos de extraversión y neuroticismo influyen de forma significativa sobre el consumo de alcohol a un nivel bajo.

Asimismo, la segunda hipótesis específica recibe respaldo empírico parcial ya que agresividad es la variable que no influye en el consumo de alcohol, aunque con una influencia insignificante, mientras que actividad sí influye de forma significativa sobre el consumo de alcohol a un nivel bajo.

\section{Tabla 2}

Análisis de regresión para rasgos de personalidad sobre consumo de alcohol

\begin{tabular}{llll}
\hline & $T$ & $P$ & $\beta$ \\
\hline Extraversión & 4.468 & $<.001$ & .305 \\
Neuroticismo & 2.059 & .041 & .156 \\
Agresividad & .425 & .671 & .032 \\
Actividad & 2.688 & .008 & .182 \\
$\mathrm{R}^{2}$ & .149 & & \\
\hline
\end{tabular}

Finalmente, y respondiendo al objetivo de investigación, se determinó que algunos rasgos de personalidad (extraversión, neuroticismo y actividad) influyen significativamente sobre el consumo de alcohol en estudiantes universitarios. 


\section{Discusión}

Los estudiantes universitarios, al ser una población vulnerable y propensa al consumo de alcohol debido a distintos factores que permiten esta actividad e influyen dentro su entorno (por ejemplo, la adaptación a diferentes círculos sociales, la exposición ante un ambiente más tolerante frente al consumo de sustancias psicoactivas, la facilidad para obtener bebidas alcohólicas en cuanto al horario variado de estudios así como la falta de supervisión de los padres o apoderados, la presión social o el hecho de querer agradar al grupo de pares (Londoño, García, Valencia \& Vinaccia, 2005), etc) pueden encontrarse en peligro en vista de las múltiples consecuencias negativas que derivan del consumo, el cual puede ser de riesgo, nocivo y de dependencia. Uno de estos factores es la personalidad, por lo que el presente estudio se enfocó en determinar la influencia existente de los cinco rasgos de personalidad (búsqueda de sensaciones, extraversión, neuroticismo, agresividad y actividad) sobre el consumo de alcohol en estudiantes universitarios de Lima. En ese sentido, los resultados apoyarán la investigación afín futura, la elaboración de recomendaciones, evaluación e intervención a futuro.

Al examinar los datos descriptivos, se encontró que la puntuación media del consumo de alcohol en estudiantes universitarios fue de 6.92, lo que indica que, en promedio, se observa un consumo de bajo riesgo en los estudiantes. Este resultado se pudo dar ya que el grupo evaluado estuvo conformado, en su mayoría, por mujeres, quienes por lo general consumen alcohol en menor medida y en menor frecuencia que los hombres (OMS, 2018; Rada \& Ispas, 2016; Ruisoto, Cacho, López-Goni, Vacab \& Jiménez, 2016).

Al tratarse de una investigación enfocada en los aspectos negativos del consumo de alcohol y su consumo disruptivo, se decidió emplear el M5FA, ya que es un modelo que demuestra representar de manera apropiada las características de personalidad tendientes a la disfuncionalidad (Aluja et al., 2007; Escorial et al., 2015; Huang et al., 2011). 
$\mathrm{Al}$ analizar la confiabilidad de la variable búsqueda de sensaciones, se halló que esta no presentó la magnitud mínima aceptable, por tal motivo, se decidió prescindir de la variable en lugar de considerarla y concluir sobre la base del error de medición, ya que el resultado sería poco confiable (Ponterotto \& Ruckdeschel, 2007).

La primera hipótesis específica planteada (los rasgos búsqueda de sensaciones, extraversión y neuroticismo influirán significativa y positivamente sobre el consumo de alcohol en estudiantes universitarios), recibe respaldo ya que se muestra una influencia significativa y positiva, a un nivel bajo, por parte de los rasgos extraversión y neuroticismo.

En cuanto al resultado de la influencia de extraversión sobre el consumo de alcohol en estudiantes universitarios, el M5FA y sus bases teóricas (Zuckerman, 2002) respaldan el hallazgo y concuerdan con que las personas con predominancia del rasgo disfrutan de la interacción y compañía de otras personas, las fiestas y reuniones grandes, actividades que, en el contexto peruano, por lo general incluyen el consumir bebidas alcohólicas (Adan et al 2017; Hakulinen et al., 2015, Marquez-Arrico \& Adan, 2013; Turiano et al., 2012).

Por motivos de la pandemia, sin embargo, las reuniones sociales se vieron interrumpidas y limitadas; no obstante, la interacción social en este caso se pudo haber dado entre miembros de una misma casa, de manera virtual o entre personas que no obedecieron las reglas del aislamiento social obligatorio y se reunieron.

En el caso de la influencia de neuroticismo sobre el consumo de alcohol, se puede entender la influencia de este rasgo sobre el consumo de alcohol en universitarios ya que al ser un grupo expuesto a distintos estresores, tanto académicos como sociales, pueden emplear el consumo de alcohol como mecanismo de afronte en búsqueda de distracción, relajamiento o generar un cambio en el estado de ánimo negativo (Adams et al., 2012; Adan et al., 2017; Marquez-Arrico \& Adan, 2013; Hicks et al., 2012, Kotov, 2010; Turiano et al., 2012). La 
situación de pandemia, a su vez, ha podido generar en los universitarios distintas emociones y sentimientos negativos, lo cual puede haber contribuido a optar por el consumo de alcohol como método de enfrentamiento ante estas circunstancias. En ese sentido, el M5FA respalda este hallazgo al tener en cuenta las bases teóricas del neuroticismo (Zuckerman, 2002).

Con respecto a la segunda hipótesis específica (los rasgos agresividad y actividad no influirán de manera significativa sobre el consumo de alcohol en estudiantes universitarios), esta recibe respaldo parcial, ya que el rasgo agresividad no demostró influencia sobre el consumo de alcohol, sin embargo, actividad, sí demostró influencia significativa a un nivel bajo.

Con relación a este hallazgo acerca de la influencia de actividad sobre consumo de alcohol, se puede entender debido a que el grupo etario estudiado, jóvenes de 18 a 29 años, pertenece a uno de los grupos que más consumen alcohol y que realiza actividades físicas de manera frecuente (Piazza-Gardner \& Barry, 2012). Asimismo, el realizar actividad física puede ser una medida de protección frente al consumo de alcohol y por este motivo, los dos factores se relacionan de manera positiva. Por motivos de la situación de pandemia y enclaustramiento, los estudiantes en quienes predomina el rasgo no han podido practicar las distintas actividades que solían realizar, lo cual incluye actividad física fuera de casa, ir personalmente a estudiar, a trabajar y en general, actividades que los mantengan ocupado. Al dificultarse la realización de dichas acciones debido a motivos de fuerza mayor, esto ha podido generar que dichas personas eleven su consumo de alcohol, lo cual explica la influencia encontrada entre el rasgo actividad y el consumo de alcohol en este grupo. En virtud de lo expuesto, el M5FA apoya lo hallado al explicar teóricamente las bases del rasgo actividad y la incomodidad de estas personas frente al aburrimiento y la necesidad de mantenerse activos (Zuckerman, 2002). 
Referente a agresividad, se determinó que el rasgo no presenta influencia sobre el consumo de alcohol. Este resultado fue el esperado ya que no se ha encontrado evidencia empírica ni teórica de una influencia entre las dos variables. Por otro lado, este hallazgo se puede explicar ya que el grupo evaluado estuvo mayormente conformado por mujeres, quienes, por lo general, presentan menor nivel del rasgo (Aluja et al., 2019)

Respecto al contraste con estudios antecedentes, los resultados hallados demuestran coincidir con distintas investigaciones (Adan et al., 2016; Hakulinen et al., 2015; NogueiraArjona et al., 2019; Motos et al., 2015; Pilatti et al., 2014; Poo \& Montes, 2010; ValdiviaLívano et al., 2018) donde se muestra que los rasgos de personalidad se relacionan con el consumo de alcohol. Por ejemplo, Poo y Montes (2010), quienes en su estudio emplearon el mismo instrumento de medición para evaluar personalidad y el cual estuvo dirigido a jóvenes entre 18 y 29 años al igual que el presente estudio, Pilatti et al. (2014), quienes, de igual manera, evaluaron estudiantes universitarios y Hakulinen et al. (2015), cuyo estudio estuvo conformado por mujeres en su mayoría, demostraron relación significativa positiva entre consumo de alcohol y extraversión y neuroticismo; Adan et al. (2016), quienes investigaron jóvenes estudiantes universitarios entre 18 a 25 años los cuales no contaban con presencia de trastornos mentales y emplearon el instrumento bajo la teoría de los M5FA para medir personalidad, hallaron influencia significativa entre consumo de alcohol y neuroticismo y Nogueira-Arjona et al., 2019, quienes a su vez analizaron estudiantes universitarios jóvenes de entre 18 a 25 años. demostraron relación positiva entre consumo de alcohol y extraversión. Asimismo, Poo y Montes (2010) encontraron asociación entre el consumo de alcohol y actividad.

Por otro lado, la investigación de Motos, Cortés, Giménez y Cadaveira (2015) determinó la existencia de una correlación nula entre consumo de alcohol, neuroticismo y extraversión. En dicha investigación, en contraste con el presente estudio, la muestra estuvo enfocada en únicamente en estudiantes de primer ciclo de la universidad. 
Por medio del presente estudio, las universidades de Lima podrán conocer el perfil de personalidad de los estudiantes y entender de qué manera este influye en el consumo de alcohol de estos. Asimismo, podrán realizar planes de prevención e intervención dirigidos a aquellos estudiantes que demuestran predominancia en cuanto a los rasgos extraversión, neuroticismo y actividad. Si es que el estado de emergencia por la pandemia continúa en el país y varias actividades siguen restringidas, este tipo de planes deberán adecuarse al contexto. Adicionalmente, las universidades podrán exponer a las personas que participarán en fiestas o eventos grandes realizadas por las mismas, ya sea de forma presencial o vía virtual, a distintos mensajes de prevención contra el consumo de alcohol de manera previa al inicio de dichas actividades.

Además, podrán brindar orientación e intervención a aquellas personas que muestran un nivel alto de neuroticismo y estén en periodo de evaluaciones o trabajos universitarios importantes, de manera que se puedan dar opciones saludables de afronte al estrés a modo de prevenir el consumo de alcohol.

De igual manera, fomentar la realización de distintas actividades motrices, en la medida de lo posible, de forma que los jóvenes puedan recuperar el estilo de vida previo a la situación de pandemia y enclaustramiento, y así, se pueda ejercer y desarrollar el rasgo actividad de manera saludable y evitar optar por el consumo de alcohol.

La principal fortaleza del estudio es ser la primera investigación en el contexto peruano que evalúa la influencia de los rasgos de personalidad sobre el consumo de alcohol empleando el modelo M5FA. Asimismo, haber estudiado las variables bajo el contexto de la pandemia por la COVID-19. Ambos motivos son considerados fortalezas ya que significa un aporte a la literatura científica y se da un mayor entendimiento en la población estudiada. 
Se reconocen ciertas limitaciones en esta investigación: teniendo en cuenta el tamaño del instrumento de medición empleado y el tiempo que requiere su ejecución, no se pudo obtener mayor muestra, la cual hubiera sido beneficioso para el estudio. Se presume que una gran cantidad de personas pudo abandonar la encuesta al haberse percatado de estos dos factores (longitud de la escala y tiempo necesario para completarla). No se pudo optimizar ni ampliar el recojo de los datos debido al corto periodo de tiempo en el cual se dio la investigación.

En conclusión, los resultados evidenciaron que algunos rasgos de personalidad (extraversión, neuroticismo y actividad) influyen sobre el consumo de alcohol en estudiantes universitarios de Lima.

Por otro lado, se recomienda emplear un instrumento de evaluación más corto para medir la variable de personalidad con el fin de poder acceder a una mayor muestra. Asimismo, replicar el estudio en estudiantes de universidades públicas de manera que se pueda ampliar la literatura en cuanto al tema investigado. Finalmente, se recomienda que el tamaño muestral sea más extenso. 


\section{Referencias}

Adams, Z. W., Kaiser, A. J., Lynam, D. R., Charnigo, R. J., \& Milich, R. (2012). Drinking motives as mediators of the impulsivity-substance use relation: Pathways for negative urgency, lack of premeditation, and sensation seeking. Addictive Behaviors, 37(7), 848-855. doi: 10.1016/j.addbeh.2012.03.016

Adan, A., Forero, D. A., \& Navarro, J. F. (2017). Personality traits related to binge drinking: a systematic review. Frontiers in Psychiatry, 8. doi:10.3389/fpsyt.2017.00134

Adan, A., Navarro, J. F., \& Forero, D. A. (2016). Personality profile of binge drinking in university students is modulated by sex. A study using the Alternative Five Factor Model. Drug and Alcohol Dependence, 165, 120-125. doi:

10.1016/j.drugalcdep.2016.05.015

Aluja, A., Cuevas, L., García, L. F., \& García, O. (2007). Zuckerman’s personality model predicts MCMI-III personality disorders. Personality and Individual Differences, 42(7), 1311-1321. doi: 10.1016/j.paid.2006.10.009

Aluja, A., Kuhlman, M., \& Zuckerman, M. (2010). Development of the ZuckermanKuhlman-Aluja Personality Questionnaire (ZKA-PQ): A factor/facet version of the Zuckerman-Kuhlman Personality Questionnaire (ZKPQ). Journal of Personality Assessment, 92(5), 416-431. doi:10.1080/00223891.2010.497406

Aluja, A., Rossier, J., García, L. F., Angleitner, A., Kuhlman, M., \& Zuckerman, M. (2006). A cross-cultural shortened form of the ZKPQ (ZKPQ-50-cc) adapted to English, French, German, and Spanish languages. Personality and Individual Differences, 41(4), 619-628. https://doi.org/10.1016/j.paid.2006.03.001 
Aluja, A., Rossier, J., Oumar, B., García, L. F., Bellaj, T., Ostendorf, F., ... Glicksohn, J. (2019). Multicultural Validation of the Zuckerman-Kuhlman-Aluja Personality Questionnaire Shortened Form (ZKA-PQ/SF) Across 18 Countries. Assessment, 107319111983177. doi:10.1177/1073191119831770

Ammar, A., Brach, M., Trabelsi, K., Chtourou, H., Boukhris, O., Masmoudi, L., ... Hoekelmann, A. (2020). Effects of COVID-19 home confinement on eating behaviour and physical activity: Results of the ECLB-COVID19 international online survey. Nutrients, 12(6). doi:10.3390/nu12061583

Aramburú, R. (2017). Sentido de vida y consumo problemático de alcohol en alumnos de universidades privadas de Lima Metropolitana (Tesis de licenciatura, Universidad de Lima, Lima, Perú). Recuperada de https://repositorio.ulima.edu.pe/handle/20.500.12724/4762

Arana, M. (2013). Factores de personalidad en estudiantes universitarios según características de consumo de alcohol (Tesis de licenciatura, Universidad Pontificia Católica del Perú, Lima, Perú). Recuperada de http://tesis.pucp.edu.pe/repositorio/handle/20.500.12404/4615

Ato, M., López-García, J. J., \& Benavente, A. (2013). Un sistema de clasificación de los diseños de investigación en psicología. Anales de Psicología, 29(3). doi:10.6018/analesps.29.3.178511

Babor, T.F., Higgins-Biddle, J.C., Saunders, J.B., \& Nonteiro, M.G. (2001). The alcohol use disorders identification test. Geneva: World Health Organization. Recuperado de https://apps.who.int/iris/bitstream/handle/10665/67205/WHO_MSD_MSB_01.6a.pdf; jsessionid $=$ DC25F0701B11C9AF4B7793748C2E7EC0 sequence $=1$ 
Ball, S. A. (2004). Personality Traits, Disorders, and Substance Abuse. In R. M. Stelmack (Ed.), On the psychobiology of personality: Essays in honor of Marvin Zuckerman (p. 203-222). Elsevier Science. doi: https://doi.org/10.1016/B978-008044209-9/50013-0

Cameron, D., Epton, T., Norman, P., Sheeran, P., Harris, P. R., Webb, T. L., ... Shah, I. (2015). A theory-based online health behaviour intervention for new university students (U@Uni:LifeGuide): results from a repeat randomized controlled trial. Trials, 16(1). doi:10.1186/s13063-015-1092-4

Cicognani, E., \& Zani, B. (2014). Alcohol use among Italian university students: The role of sensation seeking, peer group norms and self-efficacy. Journal of Alcohol and Drug Education,55(2), 17-36. Recuperado de https://www.researchgate.net/profile/Elvira_Cicognani/publication/268390043_Alcoh ol_Use_Among_Italian_University_Students_The_Role_of_Sensation_Seeking_Peer _Group_Norms_and_Self-Efficacy/links/548d4f040cf225bf66a2a0e0/Alcohol-UseAmong-Italian-University-Students-The-Role-of-Sensation-Seeking-Peer-GroupNorms-and-Self-Efficacy.pdf

Clark, L. A., \& Watson, D. (1995). Constructing validity: Basic issues in objective scale development. Psychological Assessment, 7(3), 309 - 319. Recuperado de http://www.bwgriffin.com/gsu/courses/edur9131/content/Clark_validity_scaledevelop ment.pdf

Crane, N. A., Gorka, S. M., Luan Phan, K., \& Childs, E. (2018). Amygdala-orbitofrontal functional connectivity mediates the relationship between sensation seeking and alcohol use among binge-drinking adults. Drug and Alcohol Dependence, 192, 208214 doi: 10.1016/j.drugalcdep.2018.07.044 
Cattell, R. B. (1957). Personality and motivation structure and measurement. World Book Co. Recuperado de https://psycnet.apa.org/record/1958-03918-000

Coronavirus: Perú decreta cuarentena general en el país y el cierre de fronteras durante 15 días ante la pandemia de covid-19. (16 de marzo de 2020) BBC News Mundo. Recuperado de https://www.bbc.com/mundo/noticias-america-latina-51902989

Costa, P. T., \& McCrae, R. R. (1992). The five-factor model of personality and its relevance to personality disorders. Journal of Personality Disorders, 6(4), 343 359. doi:10.1521/pedi.1992.6.4.343

Dash, G. F., Slutske, W. S., Martin, N, G., Statham, D. J., Agrawal, A. \& Lynskey, M. T. (2019). Big Five personality traits and alcohol, nicotine, cannabis, and gambling disorder comorbidity. Psychol Addict Behav, 33(4), 420-429. doi: $10.1037 / \mathrm{adb} 0000468$

Deasy, C., Coughlan, B., Pironom, J., Jourdan, D., \& Mcnamara, P. M. (2014). Psychological distress and lifestyle of students: implications for health promotion. Health Promotion International, 30(1), 77-87. doi:10.1093/heapro/dau086

Depue, R. A., \& Collins, P. F. (1999). Neurobiology of the structure of personality: Dopamine, facilitation of incentive motivation, and extraversion. Behavioral and Brain Sciences, 22(3), 491-517. doi:10.1017/s0140525x99002046

Decreto Supremo que declara Estado de Emergencia Nacional por las graves circunstancias que afectan la vida de la Nación a consecuencia del brote del COVID-19. (15 de marzo de 2020) El Peruano. Recuperado de: https://busquedas.elperuano.pe/normaslegales/decreto-supremo-que-declara-estadode-emergencia-nacional-po-decreto-supremo-n-044-2020-pcm-1864948-2/ 
Dyce, J. A. (1997). The big five factors of personality and their relationship to personality disorders. Journal of Clinical Psychology, 53(6), 587-593. doi:10.1002/(sici)10974679(199710)53:6<587::aid-jclp7>3.0.co;2-h

Escorial, S., Aluja, A., García, L. F., García, O., \& Blanch, A. (2015). Relationships between Karolinska Personality Scales and the new factors and facets of the ZuckermanKuhlman-Aluja Personality Questionnaire. Escritos de Psicología), 8(3), 20-25. https://dx.doi.org/10.5231/psy.writ.2015.2304

Eysenck, H. J. (1967). The biological basis of personality. Thomas: Spring-field. Recuperado de: https://psycnet.apa.org/record/1967-35006-000

Faul, F., Erdfelder, E., Lang, A. G., \& Buchner, A. (2007). G*Power 3: A flexible statistical power analysis program for the social, behavioral, and biomedical sciences. Behavior Research Methods, 39, 175-191. Recuperado de https://www.psychologie.hhu.de/arbeitsgruppen/allgemeine-psychologie-undarbeitspsychologie/gpower.html

Ferguson, C. J. (2009). An effect size primer: A guide for clinicians and researchers. Professional Psychology: Research and Practice, 40(5), 532-538. doi:10.1037/a0015808

Gallè, F., Sabella, E. A., Da Molin, G., De Giglio, O., Caggiano, G., Di Onofrio, V., ... Napoli, C. (2020). Understanding Knowledge and Behaviors Related to CoViD-19 Epidemic in Italian Undergraduate Students: The EPICO Study. International Journal of Environmental Research and Public Health, 17(10), 3481.

doi:10.3390/ijerph17103481 
González-Iglesias, B., Gómez-Fraguela, J., Gras, M., \& Planes, M. (2014). Búsqueda de sensaciones y consumo de alcohol: el papel mediador de la percepción de riesgos y beneficios. Anales de Psicología, 30(3), 1061-1068. doi: 10.6018/analesps.30.3.170831

Hakulinen, C., Elovainio, M., Batty, G. D., Virtanen, M., Kivimäki, M., \& Jokela, M. (2015). Personality and alcohol consumption: Pooled analysis of 72,949 adults from eight cohort studies. Drug and alcohol dependence, 151, 110-114. https://doi.org/10.1016/j.drugalcdep.2015.03.008

Herring, R., Berridge, V., \& Thom, B. (2008). Binge drinking: An exploration of a confused concept. Journal of Epidemiology and Community Health (1979-), 62(6), 476-479. Recuperado de http://www.jstor.org/stable/40665585

Hicks, B. M., Durbin, C. E., Blonigen, D. M., Iacono, W. G., \& McGue, M. (2012). Relationship between personality change and the onset and course of alcohol dependence in young adulthood. Addiction (Abingdon, England), 107(3), 540-548. https://doi.org/10.1111/j.1360-0443.2011.03617.x

Hosier, S. G., \& Cox, W. M. (2011). Personality and motivational correlates of alcohol consumption and alcohol-related problems among excessive drinking university students. Addictive Behaviors, 36(1-2), 87-94. doi: 10.1016/j.addbeh.2010.08.029

Huang, J., He, W., Chen, W., Yu, W., Chen, W., Shen, M., \& Wang, W. (2011). The Zuckerman-Kuhlman Personality Questionnaire predicts functioning styles of personality disorder: A trial in healthy subjects and personality-disorder patients. Psychiatry Research, 186(2-3), 320-325. doi: 10.1016/j.psychres.2010.07.010 
Hunt, J., \& Eisenberg, D. (2010). Mental health problems and help-seeking behavior among college students. Journal of adolescent health, 46(1), 3-10. doi:

10.1016/j.jadohealth.2009.08.008

Jobnston, L. D., O'Malley, P. M., Bacbman, J. G., \& Schulenberg, J. E. (2004). National survey results on drug use from the monitoring the future study, 1975-2003. Recuperado de https://deepblue.lib.umich.edu/bitstream/handle/2027.42/137797/vol2_2003.pdf?sequ ence $=1$

Jones, K. A., Chryssanthakis, A., \& Groom, M. J. (2014). Impulsivity and drinking motives predict problem behaviours relating to alcohol use in university students. Addictive Behaviors, 39(1), 289-296. doi: 10.1016/j.addbeh.2013.10.024

Kajonius, P., \& Mac Giolla, E. (2017). Personality traits across countries: Support for similarities rather than differences. PLOS ONE, 12(6), e0179646. doi: 10.1371/journal.pone.0179646

Kassa, G. M., Degu, G., Yitayew, M., Misganaw, W., Muche, M., Demelash, T., ... Ayehu, M. (2016). Risky Sexual Behaviors and Associated Factors among Jiga High School and Preparatory School Students, Amhara Region, Ethiopia. International Scholarly Research Notices, 2016, 1-7. doi:10.1155/2016/4315729

Kotov, R., Gamez, W., Schmidt, F., \& Watson, D. (2010). Linking "big" personality traits to anxiety, depressive, and substance use disorders: A meta-analysis. Psychological Bulletin, 136(5), 768-821. doi:10.1037/a0020327

Littlefield, A. K., \& Sher, K. J. (2010). The multiple, distinct ways that personality contributes to alcohol use disorders. Social and personality psychology compass, 4(9), 767-782. https://doi.org/10.1111/j.1751-9004.2010.00296.x 
Londoño, C., García, W., Valencia, S. C., \& Vinaccia, S. (2005). Expectativas frente al consumo de alcohol en jóvenes universitarios colombianos. Anales de Psicología, 21(2),259-267. Recuperado de: https://www.redalyc.org/articulo.oa?id=167/16721207

Loxton, N. J., Bunker, R. J., Dingle, G. A., \& Wong, V. (2015). Drinking not thinking: A prospective study of personality traits and drinking motives on alcohol consumption across the first year of university. Personality and Individual Differences, 79, 134139. doi: 10.1016/j.paid.2015.02.010

Lucas, R. E., Diener, E., Grob, A., Suh, E. M., \& Shao, L. (2000). Cross-cultural evidence for the fundamental features of extraversion. Journal of Personality and Social Psychology, 79(3), 452-468. doi:10.1037/0022-3514.79.3.452

Marquez-Arrico, J., \& Adan, A. (2013). Patología dual y rasgos de personalidad: situación actual y líneas futuras de trabajo. Adicciones, 25(3), 195-202. Recuperado de: http://www.org.adicciones.es/index.php/adicciones/article/view/46/45

Mekonen, T., Fekadu, W., Chane, T., \& Bitew, S. (2017). Problematic alcohol use among university students. Frontiers in Psychiatry, 8. doi: 10.3389/fpsyt.2017.00086

Ministerio de Salud del Perú. (2017). Situación de salud de adolescentes y jóvenes en el Perú. Recuperado de http://bvs.minsa.gob.pe/local/MINSA/4143.pdf

Motos, P., Cortés, M. T., Giménez, J. A., \& Cadaveira, F. (2015) Predictores del consumo semanal de alcohol y sus consecuencias asociadas en universitarios consumidores intensivos de alcohol. Adicciones, 27(2), 119-131. Recuperado de: https://www.adicciones.es/index.php/adicciones/article/view/700/691

Nogueira-Arjona, R., Shannon, T., Kehayes, I.-L., Sherry, S. B., Keough, M. T., \& Stewart, S. H. (2019). Drinking to keep pace: A study of the moderating influence of 
extraversion on alcohol consumption similarity in drinking buddy dyads. Addictive Behaviors, 92, 69-75. doi: 10.1016/j.addbeh.2018.12.023

Organización Mundial de la Salud. (1994). Lexicon of alcohol and drug terms. World Health Organization. Recuperado de https://apps.who.int/iris/handle/10665/39461

Organización Mundial de la Salud. (2018). Global status report on alcohol and health 2018. World Health Organization. Recuperado de https://apps.who.int/iris/handle/10665/274603.

Palacios, J. (2018). Interplay between sensation seeking and risky alcohol drinking in Mexican adolescents: An structural modeling equation approach. International Journal of Psychological Research, 11(2), 19-26. https://doi.org/10.21500/20112084.3332

Perú levanta cuarentena con 285.213 contagios y 9.677 muertos. (1 de julio de 2020) Deutsche Welle. Recuperado de: https://www.dw.com/es/per\%C3\%BA-levantacuarentena-con-285213-contagios-y-9677-muertos/a-54006409

Pilatti, A., Cupani, M., \& Pautassi, R. M. (2015). Personality and Alcohol Expectancies Discriminate Alcohol Consumption Patterns in Female College Students. Alcohol and Alcoholism, 50(4), 385-392. doi:10.1093/alcalc/agv025

Piazza-Gardner, A. K., \& Barry, A. E. (2012). Examining physical activity levels and alcohol consumption: Are people who drink more active? American Journal of Health Promotion, 26(3), 95-104. doi: 10.4278/ajhp.100929-lit-328

Pilatti, A., Urrizaga, A., Chincolla, A., \& Cupani, M. (2014). Facetas de personalidad relacionadas con el consumo elevado de alcohol en estudiantes universitarios. Perspectivas en Psicología, 11(1), 42-52. doi:10.5565/rev/qpsicologia.1247 
Ponterotto, J. G., \& Ruckdeschel, D. E. (2007). An Overview of Coefficient Alpha and a Reliability Matrix for Estimating Adequacy of Internal Consistency Coefficients with Psychological Research Measures. Perceptual and Motor Skills, 105(3), 997-1014. doi:10.2466/pms.105.3.997-1014

Poo, F. \& Montes, S. (2010). Rasgos de personalidad y patrones de consumo de alcohol en jóvenes. II Congreso Internacional de Investigación y Práctica Profesional en Psicología XVII Jornadas de Investigación Sexto Encuentro de Investigadores en Psicología del MERCOSUR. Buenos Aires: Universidad de Buenos Aires. Recuperado de: https://www.aacademica.org/000-031/279

Rada, C., \& Ispas, A. T. (2016). Alcohol consumption and accentuated personality traits among young adults in Romania: a cross-sectional study. Substance Abuse Treatment, Prevention, and Policy, 11(1). doi:10.1186/s13011-016-0080-3

Ramos-Grille, I., Gomà-i-Freixanet, M., Aragay, N., Valero, S., \& Vallès, V. (2015). Predicting treatment failure in pathological gambling: The role of personality traits. Addictive Behaviors, 43, 54-59. doi: 10.1016/j.addbeh.2014.12.010

Romero-Blanco, C., Rodríguez-Almagro, J., Onieva-Zafra, M. D., Parra-Fernández, M. L., Prado-Laguna, M., \& Hernández-Martínez, A. (2020). Physical Activity and Sedentary Lifestyle in University Students: Changes during Confinement Due to the COVID-19 Pandemic. International Journal of Environmental Research and Public Health, 17(18), 6567. doi:10.3390/ijerph17186567

Rubio, G., Bermejo, J., Caballero, M. C., \& Santo-Domingo, J. (1998). Validación de la prueba para la identificación de trastornos por uso de alcohol (AUDIT) en atención primaria. Revista Clínica Española, 198(1), 11-14. Recuperado de https://pubmed.ncbi.nlm.nih.gov/9534342/ 
Ruch, W., \& Zuckerman, M. (2001). Sensation seeking in adolescents. In: J. Raithel (Hrsg.), Risikoverhaltensweisen jugendlicher: Erklärungen, formen und prävention. Opladen: Leske + Budrich, 97-110. doi: 10.5167/uzh-77819

Ruisoto, P., Cacho, R., López-Goñi, J. J., Vaca, S., \& Jiménez, M. (2016). Prevalence and profile of alcohol consumption among university students in Ecuador. Gaceta Sanitaria, 30(5), 370-374. doi: 10.1016/j.gaceta.2016.02.008

Sáez-Francàs, N., Valero, S., Calvo, N., Gomà-i-Freixanet, M., Alegre, J., de Sevilla, T. F., \& Casas, M. (2014). Chronic fatigue syndrome and personality: A case-control study using the alternative five factor model. Psychiatry Research, 216(3), 373-378. doi: 10.1016/j.psychres.2014.02.031

Schmidt, V. (2010). Las bases biológicas del neuroticismo y la extraversión ¿Por qué nos comportamos como lo hacemos? PSIENCIA. Revista Latinoamericana de Ciencia Psicológica, 2(1),20-25. Recuperado de https://www.redalyc.org/articulo.oa?id=3331/333127086005

Silva, J. (2020) Prevalencia y factores asociados a los problemas relacionados al consumo de alcohol en estudiantes de la Universidad Nacional Mayor de San Marcos, LimaPerú, 2015 (Tesis de médico cirujano) Recuperada de https://hdl.handle.net/20.500.12672/11720

Skeel, R. L., Pilarski, C., Pytlak, K., \& Neudecker, J. (2008). Personality and performancebased measures in the prediction of alcohol use. Psychology of Addictive Behaviors, 22(3), 402-409. doi:10.1037/0893-164X.22.3.402

Son, C., Hegde, S., Smith, A., Wang, X., \& Sasangohar, F. (2020). Effects of COVID-19 on college students' mental health in the United States: Interview survey study. J Med Internet Res 22(9). doi: 10.2196/21279 
The jamovi project (2020). jamovi (Version 1.2) [Computer Software]. Recuperado de https://www.jamovi.org

Turiano, N. A., Whiteman, S. D., Hampson, S. E., Roberts, B. W., y Mroczek, D. K. (2012). Personality and Substance Use in Midlife: Conscientiousness as a Moderator and the Effects of Trait Change. Journal of research in personality, 46(3), 295-305. doi: 10.1016/j.jrp.2012.02.009

Valdivia-Lívano, S., Vega-Melgar, V., Jiménez-Meza, Y., Macedo-Poma, K., Caro-Rivera, E., Lazo-Escobar, D., Ugarte-Castillo, F., \& Mejía, C. (2018). Consumo alcohólico de riesgo en estudiantes de tres universidades de la sierra central peruana: frecuencia según sexo y factores asociados. Revista chilena de neuro-psiquiatría, 56(2), 110-116. doi: $10.4067 / \mathrm{s} 0717-92272018000200110$

Valero, S., Ramos-Quiroga, A., Gomà-i-Freixanet, M., Bosch, R., Gómez-Barros, N., Nogueira, M., ... Casas, M. (2012). Personality profile of adult ADHD: The alternative five factor model. Psychiatry Research, 198(1), 130-134. Doi :10.1016/j.psychres.2011.11.006

White, T. L. (2017). Beyond sensation seeking: a conceptual framework for individual differences in psychostimulant drug effects in healthy humans. Current Opinion in Behavioral Sciences, 13, 63-70. doi: 10.1016/j.cobeha.2016.10.008

Zuckerman, M. (1992). What is a basic factor and which factors are basic? Turtles all the way down". Personality and Individual Differences. 13 (6): 675-681. doi: 10.1016/01918869(92)90238-K

Zuckerman, M. (1994). Behavioral expressions and biological bases of sensation seeking, New York: Cambridge University Press. Recuperado de https://books.google.com/books?hl=es\&lr=\&id=ApiyY8LX5fAC\&oi=fnd\&pg=PR9\& 
$\mathrm{dq}=$ Zuckerman,+M.+(1994).+Behavioral+expressions+and+biological+bases+of + sen sation+seeking,+New+York:+Cambridge+University+Press\&ots=MUuEQ_IbiX\&sig =ogcz7shyyJ051pdDK6_FxGPGETo\#v=onepage \&q=Zuckerman\%2C\%20M.\%20(19 94).\%20Behavioral\%20expressions $\% 20$ and $\% 20$ biological $\% 20$ bases $\% 20$ of $\% 20$ sensati on\%20seeking\%2C\%20New\%20York\%3A\%20Cambridge\%20University\%20Press\& $\mathrm{f}=$ false

Zuckerman, M. (2002). Zuckerman-Kuhlman Personality Questionnaire (ZKPQ): an alternative five-factorial model. Recuperado de https://grupsderecerca.uab.cat/zkpq/sites/grupsderecerca.uab.cat.zkpq/files/zkpq7.pdf

Zuckerman, M., y Cloninger, C. R. (1996). Relationships between Cloninger's, Zuckerman's, and Eysenck's dimensions of personality. Personality and individual differences, 21(2), 283-285. doi: 10.1016/0191-8869(96)00042-6

Zuckerman, M., Kuhlman, D. M., \& Camac, C. (1988). What lies beyond E and N? Factor analyses of scales believed to measure basic dimensions of personality. Journal of Personality and Social Psychology, 54(1), 96-107. doi:10.1037/0022-3514.54.1.96

Zuckerman, M., Kuhlman, D., Joireman, J., Teta, P., \& Kraft, M. (1993). A comparison of three structural models for personality: The big three, the big five, and the alternative five. Journal of Personality and Social Psychology, 65, 757-768. Recuperado de https://grupsderecerca.uab.cat/zkpq/sites/grupsderecerca.uab.cat.zkpq/files/zkpq9.pdf 


\section{Apéndice 1}

\section{Consentimiento Informado para universitarios}

Estimado/a estudiante: La investigación titulada "Consumo de alcohol y rasgos de personalidad en jóvenes estudiantes universitarios" es conducida por Nátali Paola Arrasco Fernández, bachiller en Psicología, perteneciente a la Universidad San Ignacio de Loyola.

Este estudio se realizará en estudiantes de entre 18 a 29 años de edad, hombres y mujeres de todas las carreras, con la finalidad de identificar la relación existente entre características de personalidad y consumo de alcohol en estudiantes universitarios. Por ello, su participación es primordial en este proceso y estaremos agradecidos si accede.

La duración de toda la evaluación no será mayor a 20 minutos. Se solicitarán datos personales (sexo, edad, etc.) así como la respuesta a encuestas que valoran aspectos psicológicos (p.e., personalidad). La información recopilada se tratará en forma anónima y confidencial. Toda la información se usará para fines del estudio y sin ningún otro propósito.

El proceso es completamente voluntario y puede interrumpir su participación cuando lo considere pertinente. Si desea más información del proyecto, puede enviar un mensaje a natali.arrasco@usil.pe. En ese sentido, si usted está de acuerdo en participar, puede dar click al botón SIGUIENTE. 


\section{Apéndice 2}

Ficha Socio demográfica

\begin{tabular}{|l|l|}
\hline $\begin{array}{l}\text { Sexo } \\
\theta \text { Hombre } \\
\theta \text { Mujer }\end{array}$ & País de nacimiento: \\
Edad _ años & Lugar de residencia actual: \\
Estado civíl: & \\
\hline Carrera: & $\begin{array}{l}\text { ¿Se encuentra recibiendo tratamiento } \\
\text { psiquiátrico?: Si _ No _ _ } \\
\text { Año de ingreso a la Universidad: }\end{array}$ \\
Ciclo/semestre académico: & $\begin{array}{l}\text { alguna etapa de tu vida? } \\
\text { ingido tratamiento farmacológico en }\end{array}$ \\
\hline
\end{tabular}




\section{Apéndice 3}

\section{Cuestionario de Identificación de Trastornos debidos al Consumo de Alcohol (AUDIT)}

Marque una $\mathrm{X}$ en el cuadro de respuesta que mejor se ajuste a usted según su experiencia. Si considera que ninguna de las alternativas se ajusta de manera exacta, igualmente responda teniendo en cuenta la respuesta más adecuada para su caso.

\begin{tabular}{|c|c|c|c|c|c|}
\hline Preguntas & 0 & 1 & 2 & 3 & 4 \\
\hline $\begin{array}{l}\text { 1. ¿Con qué frecuencia consume } \\
\text { alguna bebida alcohólica (cerveza, ron, } \\
\text { pisco, vino, vodka, etc.)? }\end{array}$ & Nunca & $\begin{array}{l}\text { Una o } \\
\text { menos } \\
\text { veces al } \\
\text { mes }\end{array}$ & $\begin{array}{l}\text { De } 2 \text { a } 4 \\
\text { veces al } \\
\text { mes }\end{array}$ & $\begin{array}{l}\text { De } 2 \text { a } 3 \\
\text { veces a la } \\
\text { semana }\end{array}$ & $\begin{array}{l}4 \text { o más } \\
\text { veces a la } \\
\text { semana }\end{array}$ \\
\hline $\begin{array}{l}\text { 2. ¿Cuántos vasos de bebidas } \\
\text { alcohólicas suele tomar en un día de } \\
\text { consumo normal? }\end{array}$ & 0,1 о 2 & 3 o 4 & 5 o 6 & 7,8 o 9 & 10 o más \\
\hline $\begin{array}{l}\text { 3. ¿Con qué frecuencia toma } 6 \text { o más } \\
\text { vasos de bebidas alcohólicas en un } \\
\text { solo día? }\end{array}$ & Nunca & $\begin{array}{l}\text { Menos de } \\
\text { una vez al } \\
\text { mes }\end{array}$ & $\begin{array}{l}\text { Una vez al } \\
\text { mes }\end{array}$ & $\begin{array}{l}\text { Una vez a } \\
\text { la semana }\end{array}$ & $\begin{array}{l}\text { A diario o } \\
\text { casi a } \\
\text { diario }\end{array}$ \\
\hline $\begin{array}{l}\text { 4. ¿Con qué frecuencia, en el curso del } \\
\text { último año, le ha sido imposible parar } \\
\text { de beber una vez que había } \\
\text { empezado? }\end{array}$ & Nunca & $\begin{array}{l}\text { Menos de } \\
\text { una vez al } \\
\text { mes }\end{array}$ & $\begin{array}{l}\text { Una vez al } \\
\text { mes }\end{array}$ & $\begin{array}{l}\text { Una vez a } \\
\text { la semana }\end{array}$ & $\begin{array}{l}\text { A diario o } \\
\text { casi a } \\
\text { diario }\end{array}$ \\
\hline $\begin{array}{l}\text { 5. ¿Con qué frecuencia, en el curso del } \\
\text { último año, ha dejado de hacer lo que } \\
\text { se esperaba de usted porque había } \\
\text { bebido? }\end{array}$ & Nunca & $\begin{array}{l}\text { Menos de } \\
\text { una vez al } \\
\text { mes }\end{array}$ & $\begin{array}{l}\text { Una vez al } \\
\text { mes }\end{array}$ & $\begin{array}{l}\text { Una vez a } \\
\text { la semana }\end{array}$ & $\begin{array}{l}\text { A diario o } \\
\text { casi a } \\
\text { diario }\end{array}$ \\
\hline $\begin{array}{l}\text { 6. ¿Con qué frecuencia, en el curso del } \\
\text { último año, ha necesitado beber en la } \\
\text { mañana para sentirse mejor después de } \\
\text { haber bebido mucho el día anterior? }\end{array}$ & Nunca & $\begin{array}{l}\text { Menos de } \\
\text { una vez al } \\
\text { mes }\end{array}$ & $\begin{array}{l}\text { Una vez al } \\
\text { mes }\end{array}$ & $\begin{array}{l}\text { Una vez a } \\
\text { la semana }\end{array}$ & $\begin{array}{l}\text { A diario o } \\
\text { casi a } \\
\text { diario }\end{array}$ \\
\hline $\begin{array}{l}\text { 7. ¿Con qué frecuencia, en el curso del } \\
\text { último año, ha tenido remordimientos } \\
\text { o sentimientos de culpa después de } \\
\text { haber bebido? }\end{array}$ & Nunca & $\begin{array}{l}\text { Menos de } \\
\text { una vez al } \\
\text { mes }\end{array}$ & $\begin{array}{l}\text { Una vez al } \\
\text { mes }\end{array}$ & $\begin{array}{l}\text { Una vez a } \\
\text { la semana }\end{array}$ & $\begin{array}{l}\text { A diario o } \\
\text { casi a } \\
\text { diario }\end{array}$ \\
\hline $\begin{array}{l}\text { 8. ¿Con qué frecuencia, en el curso del } \\
\text { último año, no ha podido recordar lo } \\
\text { que sucedió la noche anterior porque } \\
\text { había estado bebiendo? }\end{array}$ & Nunca & $\begin{array}{l}\text { Menos de } \\
\text { una vez al } \\
\text { mes }\end{array}$ & $\begin{array}{l}\text { Una vez al } \\
\text { mes }\end{array}$ & $\begin{array}{l}\text { Una vez a } \\
\text { la semana }\end{array}$ & $\begin{array}{l}\text { A diario o } \\
\text { casi a } \\
\text { diario }\end{array}$ \\
\hline $\begin{array}{l}\text { 9. ¿Usted o alguna otra persona ha } \\
\text { resultado físicamente herido porque } \\
\text { usted había bebido? }\end{array}$ & No & - & $\begin{array}{l}\text { Sí, pero no } \\
\text { en el curso } \\
\text { del último } \\
\text { año }\end{array}$ & & $\begin{array}{l}\text { Sí, en el } \\
\text { último año }\end{array}$ \\
\hline
\end{tabular}




\begin{tabular}{|l|l|l|l|l|}
\hline $\begin{array}{l}\text { 10. ¿Algún familiar, amigo, médico o } \\
\text { profesional de la salud ha mostrado } \\
\text { preocupación por su consumo de } \\
\text { bebidas alcohólicas o le ha sugerido } \\
\text { que deje de beber? }\end{array}$ & & No & $\mid \begin{array}{l}\text { Sí, pero no- } \\
\text { en el curso } \\
\text { del último } \\
\text { año }\end{array}$ & $\begin{array}{l}\text { Sí, en el } \\
\text { último año }\end{array}$ \\
\hline
\end{tabular}




\section{Apéndice 4}

\section{ZKA-PQ/SF}

A continuación, se presentan varias frases que describen maneras de pensar y actuar de las personas. Por favor, indique el grado de desacuerdo o acuerdo en su caso en referencia a las frases. Si usted no ha experimentado esa circunstancia, por favor, intente describir cómo actuaría o lo que pensaría de encontrarse en esa situación. Por favor, no deje ninguna respuesta en blanco. La escala tiene 4 puntos.

\begin{tabular}{|c|c|c|c|}
\hline $\mathbf{1}$ & $\mathbf{2}$ & $\mathbf{3}$ & $\mathbf{4}$ \\
\hline $\begin{array}{c}\text { Muy en } \\
\text { desacuerdo }\end{array}$ & $\begin{array}{c}\text { Algo en } \\
\text { desacuerdo }\end{array}$ & $\begin{array}{c}\text { Algo de } \\
\text { acuerdo }\end{array}$ & $\begin{array}{c}\text { Muy de } \\
\text { acuerdo }\end{array}$ \\
\hline
\end{tabular}

Si se me provoca lo suficiente, puedo golpear a otra persona.

Me gustan algunas actividades físicas que implican algo de riesgo.

Para mí, trabajar es como una droga.

Normalmente estoy contento.

A menudo me siento intranquilo/a.

Soy muy cuidadoso/a con mis palabras y nunca devuelvo un insulto.

Me gustaría estar viajando mucho y con muchos cambios y emociones.

Me gusta mantenerme ocupado/a todo el tiempo.

Soy una persona más bien fría con los demás.

A veces me siento deprimido/a.

Me irrito muy a menudo.

Me gustan las fiestas locas y desinhibidas.

Tiendo a ser inquieto/a y a estar siempre ocupado/a con algo.

Me gusta divertir a los demás en las reuniones sociales.

Me siento desamparado/a si nadie puede aconsejarme.

Me enfado fácilmente.

Soporto mal la rutina.

Cuando yo trabajo realmente me empleo a fondo.

Soy una persona muy sociable.

Tengo poca confianza en mí mismo/a.

Es mejor que no me provoquen, pues puedo responder físicamente.

Si estuviera en el ejército, a lo mejor me presentaría voluntario/a para misiones emocionantes pero peligrosas.

Creo que cuando me jubile echaré de menos el trabajo.

Normalmente estoy de buen humor.

Me siento a menudo tenso/a sin razón aparente.

Es natural en mí soltar palabrotas cuando estoy enfadado/a.

Me gustaría viajar al extranjero dónde la gente es muy diferente a la de mi país.

Me gusta estar haciendo cosas en todo momento.

Me considero una persona seria y con dificultades para expresar mis sentimientos.

Los pensamientos negativos a veces me obsesionan. 
Si me siento atacado/a me altero y tiendo a perder los nervios.

Me gusta dejarme llevar y hacer las cosas de forma impulsiva para divertirme.

Mis amigos piensan que soy una persona inquieta o incluso algo hiperactiva.

La gente disfruta de mis ocurrencias en las conversaciones.

Pierdo la confianza en mí mismo/a cuando alguien que quiero es crítico/a conmigo.

Tengo un temperamento muy irascible.

Me gustan las situaciones que no puedes predecir.

Me esfuerzo tanto como puedo para obtener éxito en mi trabajo.

Tengo una rica vida social.

Me siento a menudo inseguro/a.

$\mathrm{Si}$ alguien me insulta tiendo a ser agresivo/a, incluyendo el uso de la fuerza.

Creo que disfrutaría trabajando de bombero/a.

Mi trabajo es mi principal placer en la vida.

Hay muchas cosas en la vida que me entusiasman agradablemente.

Soy una persona muy nerviosa.

Cuando la gente me grita, respondo gritando.

No me gustaría tener un trabajo que requiriera viajar mucho.

Desde que me despierto hasta que voy a la cama, no paro de hacer cosas.

Me cuesta demostrar afecto, incluso a mis amigos/as y familiares.

A menudo tengo ganas de llorar.

Tengo poca paciencia cuando alguien hace que me enfade.

Uno de mis objetivos principales en la vida es experimentar intensas y agradables sensaciones.

Me dicen que siempre voy con prisas a todas partes.

Me gusta hacer bromas y contar historias divertidas.

Tiendo a ser hipersensible y fácilmente me siento herido/a por los comentarios y acciones de los demás.

Me alegro cuando a ciertas personas les van mal las cosas.

Odio estar mucho tiempo haciendo lo mismo.

Me siento plenamente realizado/a en mi trabajo y me implico mucho.

Me gusta participar en actividades organizadas por mis amigos/as.

Tengo una actitud positiva hacia mí mismo/a.

Si alguien me provoca severamente, es probable que terminemos en una pelea.

Me gustaría aprender a pilotar un avión.

Probablemente no me retiraré nunca de mi trabajo.

Normalmente soy una persona avispada y alegre.

A menudo me vienen a la cabeza pensamientos sin importancia que me molestan.

Nunca digo groserías, aunque esté irritado/a.

Disfruto cuando me encuentro en situaciones nuevas en las cuales no puedes predecir cómo saldrán las cosas.

Me gusta estar activo/a desde el momento en que me levanto por la mañana.

Tengo dificultades para expresar mi cariño.

A veces me parece que me falta energía.

Controlo bien mi genio.

No reprimo mis impulsos de tener experiencias excitantes.

Tiendo a hacer las cosas rápidamente.

Los demás piensan que soy divertido/a.

Necesito ser amado/a para sentir que valgo la pena.

Tiendo a criticar a los demás, aunque a veces no me doy cuenta. 
Prefiero un trabajo imprevisible, pero divertido, que uno seguro pero rutinario. Yo rindo a plena capacidad en lo referente a mi trabajo.

Los demás me consideran solitario/a.

Pienso a menudo que los demás son mejores que yo. 